\title{
Adsorção do corante básico Verde Malaquita via carvão ativado a partir do caroço de açaí
}

\author{
Adsorption of the basic Malachite Green dye via activated carbon from the açaí seed \\ Adsorción del tinte verde de malaquita básico a través de carbón activado del semilla de açaí
}

Recebido: 11/02/2021 | Revisado: 12/02/2021 | Aceito: 16/02/2021 | Publicado: 25/02/2021

\author{
Antonio Almir Oliveira de Sousa \\ ORCID: https://orcid.org/0000-0001-5642-5890 \\ Universidade do Estado do Pará, Brasil \\ E-mail: almirsousa182@gmail.com \\ Thalia Silva Oliveira \\ ORCID: https://orcid.org/0000-0002-4327-7435 \\ Universidade do Estado do Pará, Brasil \\ E-mail: thaliao166@gmail.com \\ Luiz Eduardo Chaves Azevedo \\ ORCID: https://orcid.org/0000-0003-2199-2910 \\ Universidade do Estado do Pará, Brasil \\ E-mail: educhavesazevedo@gmail.com \\ João Rodrigo Coimbra Nobre \\ ORCID: https://orcid.org/0000-0001-6276-205X \\ Universidade do Estado do Pará, Brasil \\ E-mail: rodrigonobre@uepa.br \\ Wilson Ferando Rodrigues Stefanelli \\ ORCID: https://orcid.org/0000-0002-8524-8245 \\ Universidade do Estado do Pará, Brasil \\ E-mail: fernandostefanelli22@gmail.com \\ Thiago Antônio Paixão de Sousa Costa \\ ORCID: https://orcid.org/0000-0003-2424-8839 \\ Instituto Federal de Educação Ciência e Tecnologia do Pará, Brasil \\ E-mail: thiago.costa@ifpa.edu.br \\ João Paulo Sousa da Silva \\ ORCID: https://orcid.org/0000-0001-7174-0389 \\ Universidade do Estado do Pará, Brasil \\ E-mail: joaopaulojoao482@gmail.com \\ Ana Vitória Silva Barral \\ ORCID: https://orcid.org/0000-0002-1808-0868 \\ Universidade do Estado do Pará, Brasil \\ E-mail: anavitoriasilva675@gmail.com
}

\begin{abstract}
Resumo
Os corantes sintéticos são amplamente utilizados nos diversos seguimentos industriais, gerando um potencial poluente aos corpos hídricos. Desta forma, existindo grande necessidade de realizar-se tratamento adequado desse tipo de efluente, este estudo observou a eficiência na remoção do corante Verde Malaquita utilizando como adsorvente o Carvão Ativado (CA) produzido a partir do caroço de açaí (Euterpe oleracea), resíduo comumente gerado na região Amazônica. $\mathrm{Na}$ produção do CA foram utilizados dois agentes ativantes, Ácido Fosfórico $\left(\mathrm{H}_{3} \mathrm{PO}_{4}\right)$, sendo denominado de CAG-A e o Hidróxido de sódio $(\mathrm{NaOH})$, sendo denominado de CAG-B. A caracterização do CA foi realizada através das análises FTIR, ATG/ATD, área BET, e MEV/EDS. Para determinação da capacidade de adsorção bem como estudar e entender os mecanismos e etapas controladoras do processo de adsorção, os dados experimentais foram ajustados aos modelos matemáticos de Langmuir e Freundlich e aos modelos cinéticos de Pseudo-Primeira Ordem, Pseudo-Segunda Ordem e Difusão Intrapartícula. As análises para caracterização dos CA's indicaram a presença de grupos funcionais ácidos, carregando negativamente a superfície de CAG-A e CAG-B, favorecendo o processo de adsorção, uma vez que o adsorbato deste estudo é um corante catiônico. Os dados experimentais tanto pra CAG-A como para CAG-B melhor se ajustaram à isoterma de Freundlich, bem como também, o modelo cinético de Pseudo-Segunda Ordem teve melhor ajuste em ambos os carvões. A adsorção do Verde Malaquita em CAG-A e CAG-B mostrou-se eficiente para os CA's, obtendo-se valores de $\mathrm{q}_{\max }=113,9 \mathrm{mg} / \mathrm{g}$ e $668,88 \mathrm{mg} / \mathrm{g}$ respectivamente.
\end{abstract}

Palavras-chave: Isotermas; Cinética; Corantes. 


\begin{abstract}
Synthetic dyes are widely used in various industrial sectors, generating a potential pollutant to water bodies. Thus, with a great need to carry out an adequate treatment of this type of effluent, this study observed the efficiency in the removal of the malachite green dye using the activated carbon (AC) produced from the açaí stone (Euterpe oleracea), as an adsorbent. commonly generated in the Amazon region. In the production of AC two activating agents were used, Phosphoric Acid $\left(\mathrm{H}_{3} \mathrm{PO}_{4}\right)$, being called CAG-A and sodium hydroxide $(\mathrm{NaOH})$, being called CAG-B. The AC characterization was performed through FTIR, ATG/ATD, BET area, and SEM/EDS analyzes. To determine the adsorption capacity as well as to study and understand the mechanisms and controlling steps of the adsorption process, the experimental data were adjusted to the mathematical models of Langmuir and Freundlich and to the kinetic models of Pseudo-First Order, Pseudo-Second Order and Intraparticle Diffusion. The analysis for the characterization of the AC's indicated the presence of acid functional groups, negatively charging the surface of CAG-A and CAG-B, favoring the adsorption process, since the adsorbate in this study is a cationic dye. The experimental data for both CAG-A and CAG-B best fit the Freundlich isotherm, as well as the kinetic model of Pseudo-Second Order had better fit on both coals. The adsorption of malachite green in CAG-A and CAG-B proved to be efficient for ACs, obtaining values of qmax $=113.9 \mathrm{mg} / \mathrm{g}$ and $668.88 \mathrm{mg} / \mathrm{g}$ respectively.
\end{abstract}

Keywords: Isotherms; Kinetics; Dyes.

\title{
Resumen
}

Los tintes sintéticos se utilizan ampliamente en diversos sectores industriales, generando un potencial contaminante para los cuerpos de agua. Así, ante la gran necesidad de realizar un tratamiento adecuado de este tipo de efluentes, este estudio observó la eficiencia en la remoción del tinte Malaquita Verde utilizando el carbón activado (CA) producido a partir de la piedra de açaí (Euterpe oleracea), como adsorbente. comúnmente generada en la región amazónica. En la producción de CA se utilizaron dos agentes activantes, el Ácido Fosfórico $\left(\mathrm{H}_{3} \mathrm{PO}_{4}\right)$, que se denomina CAG-A y el Hidróxido de Sodio $(\mathrm{NaOH})$, que se denomina CAG-B. La caracterización de CA se realizó mediante análisis FTIR, ATG/ATD, área BET y MEV/EDS. Para determinar la capacidad de adsorción, así como para estudiar y comprender los mecanismos y pasos de control del proceso de adsorción, los datos experimentales se ajustaron a los modelos matemáticos de Langmuir y Freundlich y a los modelos cinéticos de Pseudo-Primer Orden, Pseudo-Segundo Orden. y difusión intrapartícula. El análisis para la caracterización de las CAs indicó la presencia de grupos funcionales ácidos, cargando negativamente la superficie de CAG-A y CAG-B, favoreciendo el proceso de adsorción, ya que el adsorbato en este estudio es un colorante catiónico. Los datos experimentales para CAG-A y CAG-B se ajustan mejor a la isoterma de Freundlich, así como el modelo cinético de Pseudo-Segundo Orden tuvo un mejor ajuste en ambos carbones. La adsorción de Verde Malaquita en CAG-A y CAG-B resultó ser eficiente para CAs, obteniendo valores de qmax = 113.9 $\mathrm{mg} / \mathrm{g}$ y $668.88 \mathrm{mg} / \mathrm{g}$ respectivamente.

Palabras clave: Isotermas; Cinética; Tintes.

\section{Introdução}

A água é um bem escasso em todo o mundo e a qualidade desta está se deteriorando a cada dia devido ao crescimento populacional, industrialização, urbanização não planejada, utilização inadequada da água etc. Atividades agrícolas e industriais intensas levaram à contaminação da água devido à liberação de grande variedade de poluentes. A falta de um tratamento adequado antes do lançamento de um efluente pode acarretar sérios danos ao meio ambiente (Giannakoudakis et al., 2018; Jain et al., 2020).

O aumento exponencial da poluição da água levou o planeta a pensar na gestão da qualidade deste recurso e, portanto, a redução de poluentes como metais, corantes etc. tornou-se uma das tarefas mais essenciais. Entre as diferentes categorias de poluição da água, os efluentes das indústrias de tingimento têm chamado significativa atenção, devido ao uso generalizado de corantes sintéticos. Esses corantes são amplamente aplicados em indústria têxtil, papel, couro etc. Sendo a indústria têxtil responsável por gerar aproximadamente $54 \%$ do efluente total de corante. Globalmente, mais de 280.000 toneladas de corantes sem tratamento são despejadas em corpos d'água (Bharti et al., 2019; Katherezan et al., 2018; Zhou et al., 2019).

A água residual colorida descarregada nos corpos hídricos receptores sem tratamento ou com tratamento insuficiente não só afeta a visão estética, mas também afeta a capacidade de atividade fotossintética do ecossistema aquático, impedindo a passagem da luz solar para os corpos d'água receptores, obstruindo o crescimento da biota aquática e causando desequilíbrio ecológico (Hui et al., 2018; Jain et al., 2020; Vikrant et al., 2018). 
Muitas vezes o tratamento convencional (físico-químico) não é eficiente na remoção de alguns tipos de corantes, pois a sua origem sintética e estrutura complexa os tornam não biodegradáveis, estáveis e, portanto, difícil tratar (Salima et al., 2013). Essas limitações podem ser solucionadas pela técnica de adsorção, devido às suas vantagens como significativa flexibilidade a uma alta carga poluente e aliada à sua eficácia, versatilidade, possibilidade de reutilização do adsorvente, baixo custo com energia consumida em comparação com outros métodos de tratamento, além da disponibilidade de uma ampla gama de adsorventes (Saleth et al., 2017; Saleth et al., 2018).

$\mathrm{O}$ açaizeiro - palmeira que produz o fruto açaí, possui grande destaque em toda a Amazônia em detrimento aos demais recursos vegetais devido principalmente à sua opulência e por se tratar de um importante alimento para as populações locais, além disso, constitui-se como principal insumo na produção de palmitos para a agroindústria brasileira (Oliveira et al., 2016). Segundo o Instituto Brasileiro de Geografia e Estatística - IBGE (2020) o Estado do Pará é o maior produtor nacional de açaí, com uma produção anual de 1.320.150 toneladas de frutos.

De acordo com Sato (2018) somente é utilizado $17 \%$ do caroço de açaí para a extração da poupa, gerando $83 \%$ de resíduos. Desta forma, observa-se que a quantidade de material gerado pode se tornar um potencial impacto ambiental, sendo necessários estudos e pesquisas com intuito de reaproveitar esses resíduos a fim de minimizar os impactos causados pela deposição irregular dos caroços de açaí no Meio Ambiente.

\section{Fundamento Teórico}

\subsection{Efluentes industriais com corantes}

Os corantes sintéticos são amplamente utilizados nas indústrias têxtil, farmacêutica, alimentícia, cosmética, de couro, papel e outras. Os efluentes dessas indústrias são altamente coloridos e, dentre todos os poluentes são uma das classes mais importantes que representam problemas ambientais na água (Konicki et al., 2015).

A água residual de tingimento é caracteristicamente alta em conteúdo inorgânico, alto teor de sal e baixa biodegradação. A descarga direta de efluentes de corantes dessas indústrias pode causar sérios problemas ao meio ambiente e à saúde humana pois podem ser tóxicos, cancerígenos, contribuem com carga orgânica ao meio ambiente e são a fonte de poluição estética relacionada à cor, o que também implica na dificuldade de penetração da luz nos ecossistemas aquáticos. Consequentemente, a eliminação da cor dos efluentes de tingimento é uma das principais preocupações ambientais (Ahmad et al., 2015).

Os corantes são resistentes a muitos tratamentos convencionais de água devido à sua estrutura química complexa e baixa biodegradabilidade (De Souza et al., 2019). Nesse sentido muitos tratamentos e tecnologias foram investigadas e aplicadas, como separação por membrana, adsorção, filtração e troca iônica. Dentre esses, a adsorção em superfície de carvão ativado tem se mostrado um dos métodos mais eficientes na remoção de orgânicos e poluentes inorgânicos de águas residuais devido à disponibilidade de diferentes adsorventes, baixo custo e alta eficiência comparada as demais para a descoloração de águas industriais (Hao et al., 2018).

\subsection{Carvão ativado}

O CA é obtido a partir de materiais carbonosos com estrutura cristalográfica desorganizada, constituída por microcristais distribuídos aleatoriamente. Esses materiais apresentam uma alta área de superfície específica (500-1500 m²/g), uma ampla variedade de grupos funcionais (carboxilatos, carbonilas, hidroxilas, aminas) e uma distribuição de tamanho de poro ( $<1-100$ $\mathrm{nm}$ ). Essas propriedades dão ao CA uma capacidade extraordinária de adsorver grande diversidade de moléculas (Masoumi \& Dalai, 2020; Pallarés \& Araúzo, 2018). Se caracteriza por ser um material carbonáceo, com estrutura porosa e grande área superficial desenvolvida, que permite uma adsorção eficiente (Nascimento, 2019). 
Os CA's têm sido extensivamente estudados ao longo dos anos por muitos pesquisadores em todo o mundo devido à sua estrutura química, características texturais e, sua alta propriedade adsortiva para uma variedade de substâncias orgânicas e inorgânicas. Os CA's são, portanto, materiais altamente valiosos como adsorventes eficientes para a remoção de uma variedade de poluentes, como corantes, metais pesados, e pesticidas (Junior et al., 2014).

No Brasil, uma matéria-prima viável para a produção de CA é o caroço de açaí (Euterpe Oleracea), devido sua abundância na região norte, alto teor de carbono em sua composição, rico em celulose, hemicelulose e lignina e ótimo desempenho econômico, social e cultural de importância na Amazônia brasileira (Sato, 2018). A semente de açaí é um fruto que cresce nas palmeiras nativas brasileira na região Amazônica, seu resíduo é muito produzido por estabelecimentos comerciais que, após a extração do suco de açaí criam descartes inadequados e problemas de poluição devido ao seu grande volume gerado (De Souza et al., 2019; de Lima Yamaguchi et al., 2015).

\subsection{Tipos de ativação}

As características texturais e químicas dos CA's estão diretamente relacionadas ao precursor selecionado e às condições utilizadas durante os procedimentos de síntese. As altas taxas de adsorção dos CA's se devem à funcionalização de suas superfícies, que melhora as interações adsorvente-adsorbato, e à estrutura de poros bem desenvolvida, que permite acesso aos sítios de adsorção internos. Os procedimentos de síntese de CA consistem basicamente em uma etapa de carbonização do material precursor sob um fluxo de gás inerte, seguida de uma etapa de ativação física ou química, que promove o desenvolvimento da porosidade do material carbonizado (Bedin et al., 2016).

A ativação física consiste na carbonização por meio de pirólise com uso de $\mathrm{CO}_{2}$ ou vapor de água, neste processo ocorre a remoção de materiais que não contêm carbono, seguido de exposição a temperaturas que variam de $800^{\circ} \mathrm{C}$ a $1000^{\circ} \mathrm{C}$ (Rimoli et al, 2019). Já na ativação química, uma matéria-prima é impregnada com um elemento ativador como $\mathrm{H}_{3} \mathrm{PO}_{4}, \mathrm{ZnCl}_{2}, \mathrm{NaOH}$, $\mathrm{KOH}$, entre outros, e o material impregnado é aquecido em atmosfera inerte. A ativação química é preferível à ativação física devido ao maior rendimento, simplicidade, menor temperatura e menor tempo de ativação e bom desenvolvimento da estrutura porosa. Assim sendo, o processo de ativação química tem sido amplamente utilizado por muitos pesquisadores para a preparação de carvões ativados de diferentes resíduos orgânicos (Ahmed \& Dhedan, 2012).

\subsection{Processo de adsorção e fatores que influenciam}

A adsorção pode ser entendida como uma transferência de massa do tipo sólido - fluido, onde é avaliada a habilidade de determinados sólidos em reter na sua superfície os poluentes presentes nas substâncias líquidas ou gasosas (Nascimento et al. 2014), isto é, a acumulação de compostos orgânicos ou inorgânicos (adsorbato) na superfície do material adsorvente.

As moléculas presentes no componente fluído, ao tocar o sólido, se espalham por toda a sua interface devido a presença de forças atrativas não compensadas na superfície do adsorvente (Galindo, 2012), ou seja, quando as moléculas do adsorbato entram em contato com o adsorvente, cria-se um campo de forças em virtude do desequilíbrio das forças atuantes na superfície do sólido, atraindo e retendo essas moléculas nos sítios de adsorção.

A adsorção, dependendo da natureza das forças envolvidas, se dará por meio de dois principais fenômenos: fisissorção e quimissorção. De acordo com Mangueira (2014), no processo de adsorção física, a interação entre o adsorbato e a superfície do adsorvente é relativamente fraca, associada principalmente às Forças de Van Der Waals ou forças eletrostáticas, que permitem a formação de várias camadas do soluto nos sítios ativos do adsorvente. Na adsorção química, as moléculas do adsorbato trocam ou partilham seus elétrons com a superfície do adsorvente, dando ao processo características de uma reação química, formando assim ligações extremamente fortes (Azevedo, 2015; Nascimento et al., 2014). 
A adsorção é um processo dependente das características físicas e químicas da solução e do adsorvente. Desta forma, são vários os fatores que influenciam nesse processo, tais como: as características do adsorbato, propriedades do adsorvente (a saber área superficial, tamanho e distribuição dos poros, grupos funcionais constantes na sua superfície) e condições operacionais (especialmente, temperatura e pH) (Nascimento et al., 2014).

Devido ao fato de a adsorção ser um processo que ocorre na superfície, a Área Superficial Específica (ASE) está diretamente ligada à intensidade do fenômeno adsortivo (Nascimento et al., 2014). Vale ressaltar também que a área superficial pode aumentar ou diminuir utilizando tratamentos oxidantes, de acordo com a natureza de cada material precursor, de forma que segundo Guimarães (2006) geralmente a oxidação aumenta enquanto a redução diminui a ASE.

\subsection{Estudos de adsorção e ajustes aos modelos matemáticos}

Uma isoterma de adsorção relaciona a quantidade de gás adsorvido num adsorvente (sólido) com pressão de equilíbrio a uma temperatura constante, para padronizar a análise de diferentes pares de adsorção. Os tipos mais comuns de isotermas de adsorção, segundo a classificação da IUPAC (Muttakin et al., 2018), encontram-se na Figura 1.

Figura 1 - Tipos de isotermas de acordo com a classificação da IUPAC.

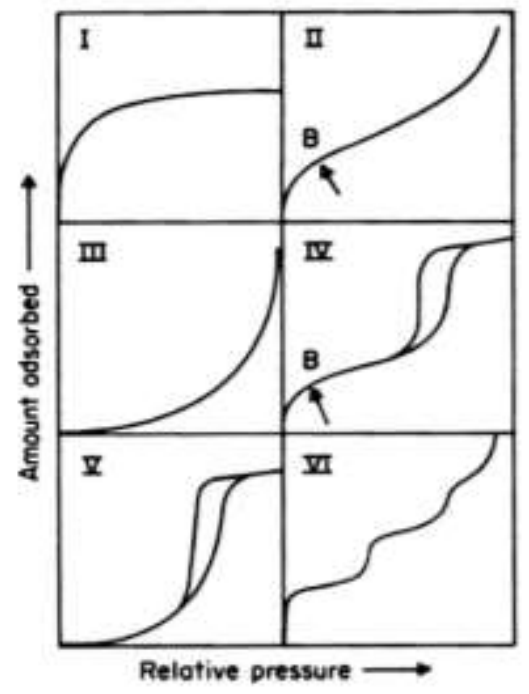

Fonte: Muttakin et al., 2018.

De acordo com Muttakin et al. (2018) a isoterma do tipo I é caracterizada por adsorção em monocamada. A absorção mostra um rápido aumento com a pressão até atingir um platô a uma pressão suficientemente alta. Este reversível tipo isotérmico pode ser obtido para sólidos microporosos com superfícies externas relativamente pequenas. O limite de captação depende do volume de microporos acessíveis. A isoterma do tipo II exibe adsorção de multicamadas e, portanto, o platô de captação observado na isoterma do tipo I está ausente. A aceitação continua a aumentar mesmo quando a pressão relativa está perto da unidade. Este tipo também é reversível e tipicamente macroporoso. Isoterma III, este também é um tipo reversível de isoterma e a curva de captação é convexo à razão de pressão em toda a faixa. A forma característica da isoterma mostra baixos teores de umidade em atividades de baixa água e a absorção aumenta acentuadamente em atividades de alta água. A isoterma do tipo IV é caracterizada por seu ciclo de histerese que é atribuída ao enchimento / desocupação dos mesoporos por condensação capilar. O ramo inferior do loop de histerese representa medição por adição progressiva de gás do adsorvente e o ramo superior representa a medição por retirada progressiva. Este tipo pode ser considerado uma combinação de isotermas de adsorção de Tipo I e Tipo 
II. A aceitação aumenta bastante com pressão dentro da região de baixa pressão e então sofre adsorção de monocamada a multicamadas. A condensação no mesopóro domina quando a pressão se aproxima da saturação pressão.

Outra classificação bastante usual para as isotermas é a classificação de Giles, que de acordo com Azevedo (2015), a isoterma do tipo C (figura 2a) é uma curva que significa que a razão entre a concentração de composto e adsorvido no sólido é o mesmo em qualquer concentração. A isoterma do tipo do L (figura $2 b$ revela que a razão entre a concentração do composto na solução e adsorvido no sólido diminui quando a concentração de soluto aumenta. O tipo H (Figura 2c) é um caso particular, onde a inclinação inicial é muito alta, onde o composto exibe uma afinidade tão alta para o sólido que a inclinação inicial não pode ser distinguida do infinito e por último, na isoterma do $\mathrm{S}$ (Figura 2d) a curva é sigmoidal e, portanto, tem um ponto de inflexão. Este tipo de isoterma é sempre o resultado de pelo menos dois mecanismos opostos.

Figura 2 - Classificação das isotermas segundo Giles.

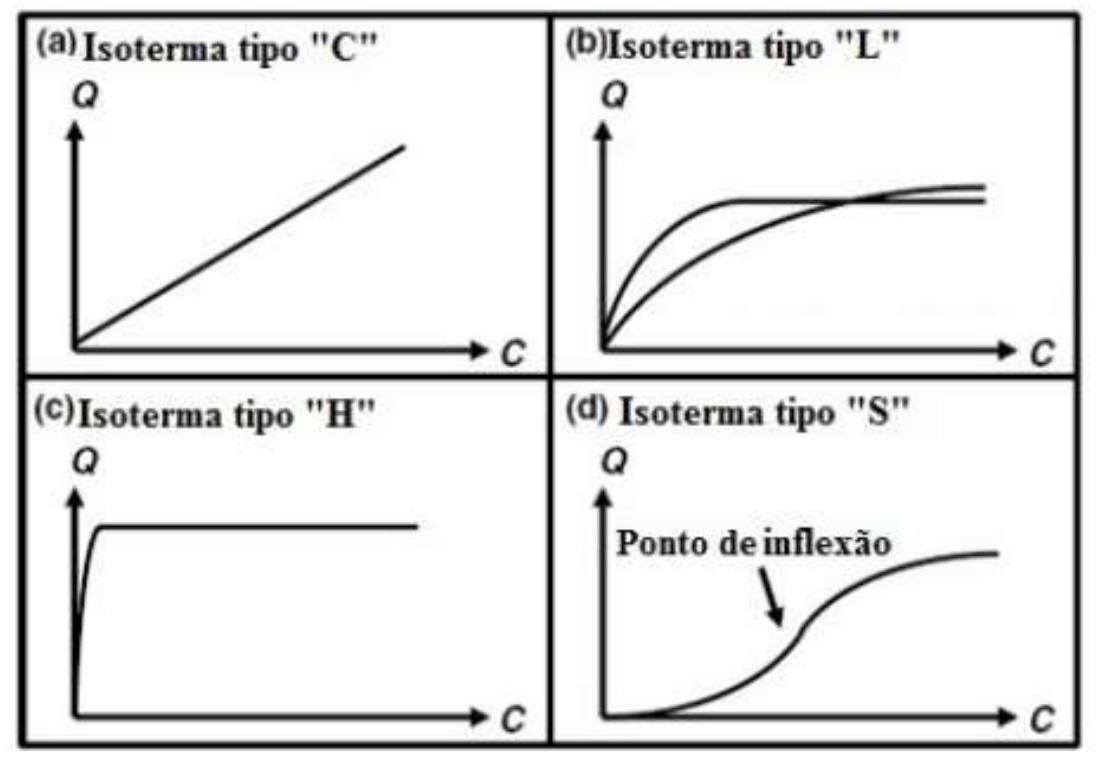

Fonte: Azevedo (2015).

Em algumas isotermas observou-se que o fenômeno de dessorção não coincide com a adsorção. Essa mudança de trajetória na dessorção, chamada de histerese tem diferentes formas que correspondem as diferentes geometrias que os poros podem assumir. A ocorrência de histerese está relacionada às características da estrutura porosa e ao mecanismo de adsorção (Marinho et al., 2019). A classificação segundo a IUPAC para os quatro tipos de histerese designados, como H1, H2, H3 e H4 são representados na Figura 3.

Figura 3 - Tipos de histerese presentes tipicamente nas isotermas de adsorção.

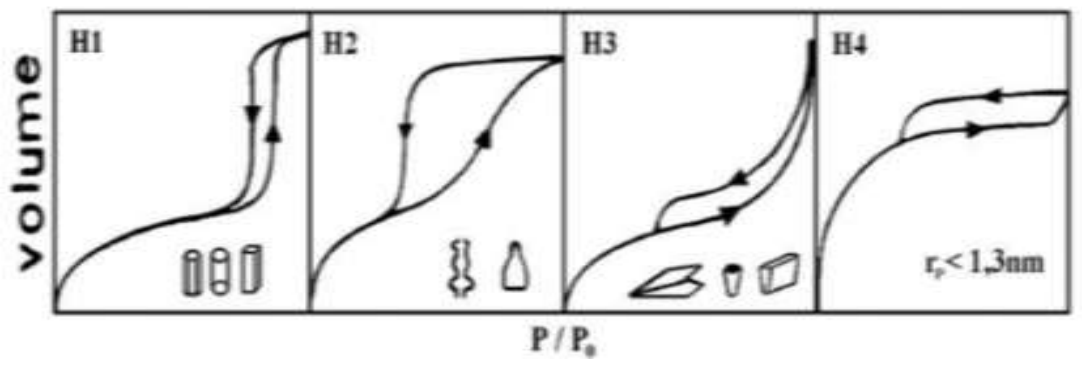

Fonte: Almeida (2015). 
O fenômeno da histerese pode ser identificado em quatro tipos: A histerese tipo H1 é associada a materiais porosos constituídos por aglomerados rígidos de partículas esféricas de tamanho uniforme, histerese tipo H2 está relacionada a poros com gargalo estreito e corpo largo, a do tipo H3 está associada a poros com formato de cunha, cone ou placas paralelas, enquanto a do tipo $\mathrm{H} 4$ pode ser relacionada a poros estreitos tipo cunha e cone (Almeida, 2015).

As isotermas de adsorção auxiliam na predição do mecanismo de adsorção e nas interações entre o adsorvente e o adsorbato durante o processo de adsorção, a temperatura constante e pH específico da solução. A quantidade de substância adsorvida no adsorvente é calculada com base na concentração restante (principalmente em mg/g) na solução (Ray, 2020). Desta forma, a equação geral da capacidade máxima de adsorção é dada pela equação (1):

$$
q_{e}=\frac{\left(C_{0}-C_{e}\right) V}{m}
$$

onde,

qe = capacidade de adsorção;

$\mathrm{Co}=$ concentração inicial;

$\mathrm{Ce}=$ concentração final;

$\mathrm{V}=$ volume; $\mathrm{e}$

$\mathrm{M}=$ massa do adsorvente.

Segundo Ray (2020), cada isoterma de adsorção possui equações matemáticas para calcular os outros parâmetros, como capacidade de adsorção, e para prever o mecanismo. Com base na concentração inicial $\left(\mathrm{C}_{\mathrm{o}}\right)$, no tempo t e na concentração final no equilíbrio (Ce) do corante na solução, os valores são colocados nas respectivas equações matemáticas e o modelo mais adequado fornece informações sobre o mecanismo de adsorção e propriedades da superfície do material adsorvente. Ainda segundo o mesmo autor, vários modelos de adsorção, incluindo as isotermas Henry, Langmuir, Dubinin Radus kevich, Freundlich, Temkin, Redlich-Peterson, Koble Corrigan, Jovanovic e Halsey, foram desenvolvidos para ajustar dados experimentais para prever o mecanismo de adsorção (monocamada / multicamadas ou homogêneos / heterogêneos).

Os modelos de isotérmica de adsorção mais usados para a remoção de contaminantes das águas residuais são as isotérmicas de adsorção Langmuir e Freundlich, que ajudam a calcular a capacidade máxima de adsorção $\left(\mathrm{Q}_{\mathrm{m}}\right)$ dos adsorventes. Todas as isotermas são baseadas em equações matemáticas não lineares que podem ser transformadas em modelos lineares $(\mathrm{y}=$ $a x+b)$, a qual uma linha reta é observada no gráfico. $O$ valor da constante de regressão linear $\left(R^{2}\right)$ ajuda a descobrir qual isotérmica de adsorção é adequado para explicar o comportamento de adsorção (Kumar et al., 2016).

$\mathrm{O}$ valor de $\mathrm{R}^{2}$ próximo à unidade promove a acessibilidade do modelo isotérmico para estudar a adsorção. $\mathrm{O} \mathrm{R}^{2}$ é a ferramenta mais confiável para definir o modelo mais adequado ao analisar o sistema de adsorção, quantificar a distribuição do adsorvido e verificar as premissas teóricas da isotérmica de adsorção (Ray et al., 2020).

\subsubsection{Isotermas de Langmuir}

Este é o modelo de isotérmica de adsorção mais estudado regularmente, com base no pressuposto da formação de uma monocamada de adsorbato homogeneamente no adsorvente. O processo de adsorção que segue esse modelo isotérmico de Langmuir indica que o adsorvente usado exibe números finitos de locais ativos idênticos disponíveis para a interação com o adsorbato e não há interação lateral devido ao impedimento estérico entre as moléculas adsorvidas na superfície. (Mittal \& Mishra, 2014).

Portanto, ocorre uma distribuição homogênea do adsorvido na superfície do adsorvente, com constante entalpia e energia de ativação por adsorção (Kundu \& Gupta, 2006). Para Kumar et al. (2017), uma vez que todos os locais ativos da 
superfície disponíveis sejam ocupados pela molécula/íon adsorvido, não haverá mais adsorção na superfície. A representação matemática da isoterma de adsorção de Langmuir é expressa como:

$$
Q_{e}=\frac{Q_{m} \cdot K_{L} \cdot C_{e}}{1+K_{L} \cdot C_{e}}
$$

onde,

$\mathrm{C}_{\mathrm{e}}=$ representa a quantidade de adsorvido restante na solução em equilíbrio ( $\left.\mathrm{mg} / \mathrm{g}\right)$;

$\mathrm{Q}_{\mathrm{e}}=$ representa a quantidade de adsorbato adsorvido por unidade de adsorvente $(\mathrm{mg} / \mathrm{g})$;

$\mathrm{Q}_{\mathrm{m}}=$ significa a capacidade máxima de adsorção $(\mathrm{mg} / \mathrm{g}) ; \mathrm{e}$

$\mathrm{K}_{\mathrm{L}}=$ é a constante isotérmica de Langmuir $(\mathrm{L} / \mathrm{mg})$.

$\mathrm{O}$ valor de $\mathrm{K}_{\mathrm{L}}$ se correlaciona com a variação nas características da superfície do adsorvente, como área superficial específica e porosidade. A eq. (3) pode ser rearranjada para a sua forma linear para calcular os parâmetros isotérmicos de Langmuir. Desta forma, temos:

$$
\frac{C_{e}}{Q_{e}}=\frac{1}{Q_{m}} \cdot C_{e}+\frac{1}{Q_{m} \cdot K_{L}}
$$

O gráfico isotérmico de Langmuir é plotado por $\mathrm{C}_{\mathrm{e}} / \mathrm{Q}_{\mathrm{e}}$ (eixo $\mathrm{Y}$ ) versus $\mathrm{C}_{\mathrm{e}}$ (eixo $\mathrm{X}$ ) e uma linha reta é observada com os valores de interceptação $1 /\left(\mathrm{Q}_{\mathrm{m}} \cdot \mathrm{K}_{\mathrm{L}}\right)$ e inclinação $1 / \mathrm{Q}_{\mathrm{m}}$. $\mathrm{O}$ valor da constante de regressão $\left(\mathrm{R}^{2}\right)$ do gráfico justifica a aplicabilidade deste modelo. O gráfico de Langmuir também ajuda a calcular a constante adimensional, que é conhecida como fator de separação $\left(R_{L}\right)$, a saber:

$$
R_{L}=\frac{1}{1+K_{L} \cdot C_{o}}
$$

$O$ valor de $R_{L}$ indica se o método de adsorção é favorável $\left(0<R_{L}<1\right)$ ou desfavorável $\left(R_{L}>1\right)$, irreversível $\left(R_{L}=0\right)$ ou linear $\left(\mathrm{R}_{\mathrm{L}}<1\right)$ usando $\mathrm{K}_{\mathrm{L}}$ e a concentração inicial $\left(\mathrm{C}_{\mathrm{o}}\right)$ em $\mathrm{mg} / \mathrm{g}$ de adsorbato na solução. $\mathrm{O}$ comportamento de adsorção de vários corantes, íons de metais pesados e outros poluentes da água pode ser explicado seguindo o sistema de modelos isotérmicos de adsorção de Langmuir (Santhosh et al., 2016).

\subsubsection{Isotermas de Freundlich}

A isotérmica de Freundlich é altamente aceita para sistemas de adsorção heterogêneos nos quais o adsorvente exibe locais de superfície ativos diferentes com distribuição não uniforme de energias (Kumar et al., 2016; Kumar et al., 2017). Esta isotérmica não se limita à construção de uma monocamada de adsorbato em adsorvente, mas segue uma tendência de multicamadas. A representação matemática da isoterma de adsorção de Freundlich pode ser escrita da seguinte maneira:

$$
Q_{e}=K_{f} \cdot\left(C_{e}\right)^{\frac{1}{n}}
$$

Modificando para sua forma linear, a expressão pode ser reescrita como eq. (6):

$$
\log Q_{e}=\log K_{f}+\frac{1}{n} \log C_{e}
$$

onde,

$\mathrm{Q}_{\mathrm{e}}=$ significa a quantidade de adsorbato adsorvido no adsorvente em equilíbrio (mg/g);

$\mathrm{C}_{\mathrm{e}}=$ representa a quantidade de adsorbato restante na solução em equilíbrio $(\mathrm{mg} / \mathrm{g})$;

$\mathrm{K}_{\mathrm{f}}=$ é a capacidade de adsorção do adsorvente $\left(\mathrm{mg} / \mathrm{g}(\mathrm{L} / \mathrm{mg})^{1 / n}\right)$; e

$\mathrm{n}=$ significa a intensidade de adsorção.

O gráfico de adsorção de Freundlich é plotado entre o $\log Q_{\mathrm{e}}$ e o $\log \mathrm{C}_{\mathrm{e}}$ para ajudar a calcular a capacidade de adsorção e a intensidade de adsorção usando interceptação e inclinação, respectivamente. Um valor mais alto de $1 / \mathrm{n}(1 / \mathrm{n}>1)$ indica que 
o adsorvente funciona bem para soluções de alta concentração; no entanto, o baixo valor de $1 / \mathrm{n}(1 / \mathrm{n}<1)$ mostra o potencial da capacidade de adsorção de adsorvente para soluções de baixa concentração (Yan et al., 2012). Geralmente, materiais em multicamadas ou materiais híbridos seguem a isoterma de adsorção de Freundlich, pois um modelo intra-estrutura diferente em materiais híbridos restringe a formação de uma monocamada idêntica de soluto na superfície (Ayawei et al., 2017).

\subsection{Cinética de adsorção e ajuste aos modelos cinéticos}

Estudos de cinética de adsorção são importantes no tratamento de efluentes aquosos porque fornecem informações valiosas sobre o mecanismo do processo de adsorção (Prola et al., 2013; Royer et al., 2010). A cinética é dependente de fatores materiais, como os tipos de adsorvente e adsorbato, e de fatores experimentais, como temperatura e pH (Slimani et al., 2011; Regti et al., 2017). É importante garantir condições experimentais constantes durante a adsorção (Tan \& Hameed, 2017). Naushad et al. (2014) cita vários modelos cinéticos que podem ser usados para descrever a cinética de adsorção, dentre os quais estão, modelo de pseudo-primeira ordem, pseudo-segunda ordem e modelos de difusão intrapartícula.

A equação de pseudo-primeira ordem (PPO) foi proposta por Largergren em 1898 para adsorção de ácido oxálico e malônico em carvão ativado (Dhedan \& Ahmed, 2012). A forma diferencial nesse modelo é representada pela equação (7):

$$
\frac{d q}{d t}=k_{1}\left(q_{e}-q\right)
$$

onde:

$\mathrm{q}_{\mathrm{e}}=$ Quantidade de soluto adsorvido no equilíbrio $(\mathrm{mg} / \mathrm{g})$;

$\mathrm{q}_{\mathrm{t}}=$ Quantidade de soluto adsorvido no tempo $\mathrm{t}(\mathrm{mg} / \mathrm{g})$;

$\mathrm{k}_{1}=$ Contante de velocidade $\left(\mathrm{min}^{-1}\right)$;

$\mathrm{t}=$ Tempo (min).

Integrando a eq. (7) com as condições iniciais $q=0$, quando $t=0$, temos;

$$
q=q_{e}(1-\exp (-k t))
$$

Assim, a equação linear deste modelo pode ser representada da seguinte forma:

$$
\ln \left(\frac{q_{e}}{q_{e}-q}\right)=k_{1} t
$$

A plotagem de $\ln \left(\left(\mathrm{q}_{\mathrm{e}}-\mathrm{q}\right) / \mathrm{q}_{\mathrm{e}}\right)$ versus $\mathrm{t}$ resulta uma linha reta que passa pela origem com uma inclinação $\mathrm{k}_{1}$ para sistemas que obedecem a este modelo (Tan \& Hameed, 2017). A constante de velocidade $k_{1}$ é uma função das condições do processo, ou seja, diminui com o aumento da concentração inicial em massa, que pode ser entendido da seguinte forma: $1 / \mathrm{k}_{1}$ é a escala de tempo para o processo atingir o equilíbrio. É necessário um tempo maior (menor $\mathrm{k}_{1}$ ) se a concentração inicial $\left(\mathrm{C}_{0}\right)$ for maior. Sabe-se também que $\mathrm{k}_{1}$ pode ser uma função crescente de $\mathrm{C}_{0}$ ou independente de $\mathrm{C}_{0}$ (Yousef, El-Eswed, \& Al-Muhtaseb, 2011).

Neste modelo, o ideal é que $\mathrm{k}_{1}$ seja influenciado por condições experimentais, como $\mathrm{pH}$ e temperatura. Como o comportamento de equilíbrio (isotermas) é afetado por esses dois fatores, isolar seus efeitos para o valor de $\mathrm{k}_{1}$ seria experimentalmente difícil. Assim, o tamanho de partícula pequeno está associado a grandes valores de $\mathrm{k}_{1}$ (Mishra \& Biswas, 2015; Vicent et al. 2014). O modelo de Pseudo Primeira Ordem é considerado válido para longos tempos de adsorção quando o sistema está próximo do equilíbrio, além disso, o PPO também demonstra ser válido apenas na fase inicial de adsorção (Tan \& Hameed, 2017).

O modelo de pseudo-segunda ordem (PSO) tem a vantagem de investigar a cinética de adsorção para soluções de baixa concentração. Supõe-se que em uma reação de pseudo-segunda ordem, a etapa limitante da taxa é a quimissorção e a capacidade de adsorção depende principalmente dos sítios de superfície ativos no adsorvente. A equação diferencial para o modelo de pseudo-segunda ordem é representada como (Ray et al., 2020): 


$$
\frac{\mathrm{dq}}{\mathrm{dt}}=\mathrm{k}_{2}\left(\mathrm{q}_{\mathrm{e}}-\mathrm{q}\right)^{2}
$$

onde,

$\mathrm{k}_{2}=$ constante de taxa de pseudo-segunda ordem.

$\mathrm{q}_{\mathrm{e}}=$ Quantidade de soluto adsorvido no equilíbrio $(\mathrm{mg} / \mathrm{g})$;

$\mathrm{q}_{\mathrm{t}}=$ Quantidade de soluto adsorvido no tempo $\mathrm{t}(\mathrm{mg} / \mathrm{g})$;

$\mathrm{t}=$ Tempo $(\min )$.

Integrando a Eq. (10) com condições iniciais de $\mathrm{q}=0$ quando $\mathrm{t}=0$ e o rearranjo subsequente obtém a forma não linear (De Souza, 2019):

$$
\mathrm{q}_{\mathrm{t}}=\frac{\mathrm{k}_{2} \mathrm{q}_{\mathrm{e}}^{2} \mathrm{t}}{1+\left(\mathrm{q}_{\mathrm{e}} \mathrm{k}_{2} \mathrm{t}\right)}
$$

Os efeitos do $\mathrm{pH}$ e da temperatura no $\mathrm{k}_{2}$ não são bem estudados devido às dificuldades que surgem dos efeitos nas formas de isotermas de equilíbrio. $\mathrm{O}$ tamanho de partícula pequeno produz um valor maior de $\mathrm{k}_{2}$ devido à redução da resistência à difusão intrapartícula (Vicent et al., 2014).

Azevedo (2015) representa o modelo matemático de difusão intrapartícula na equação seguinte:

$$
q_{e}=\mathrm{k}_{\mathrm{i}} \mathrm{t}^{1 / 2}+\mathrm{C}
$$

onde,

$\mathrm{k}_{\mathrm{i}}=$ é a constante da taxa de difusão intrapartícula $\left(\mathrm{mg} / \mathrm{g} \cdot \mathrm{min}^{1 / 2}\right) ; \mathrm{e}$

$\mathrm{C}=$ é a constante de espessura da camada limite ( $\mathrm{mg} / \mathrm{g})$.

Quando ocorre a difusão intrapartícula dizemos então que qt versus $\mathrm{t}^{1 / 2}$ é linear e se o gráfico passa pela origem, então o processo de limitação de taxa é devido apenas à difusão intrapartícula. Caso contrário, algum outro mecanismo junto com a difusão intrapartícula também está envolvido (Xu, Zhou \& Huang 2008; Naushad et al., 2015).

\section{Metodologia}

O estudo possui caráter experimental concedendo o detalhamento dos principais processos bem como equipamentos e instrumentos utilizados na coleta e processamento de dados com intuito de oferecer uma visão geral do trabalho (Köche, 2011), além disso, esta pesquisa aborda análises quali-quanti, onde as análises qualitativas irão complementar os resultados numéricos (Pereira, Shitsuka, Parreira \& Shitsuka, 2018)

\subsection{Produção do carvão ativado}

O material precursor utilizado para este estudo, foi o caroço do açaí, coletado em pontos de venda da polpa na mesma cidade. Após a coleta lavou-se os caroços com água corrente e em seguida foi realizada a secagem na estufa por 24 horas a 102 ${ }^{\circ} \mathrm{C}( \pm 3)$. A ativação química foi realizada utilizando duas soluções ativantes: uma ácida com ácido fosfórico $\left(\mathrm{H}_{3} \mathrm{PO}_{4}\right)$, sendo denominado Carvão Ativado Granular Ácido (CAG-A) e outra ativação básica, com hidróxido de sódio (NaOH) - sendo denominado Carvão Ativado Granular Básico (CAG-B).

$\mathrm{Na}$ ativação ácida, foram mergulhados $500 \mathrm{~g}$ do material precursor em 1 litro de solução de $\mathrm{H}_{3} \mathrm{PO}_{4}$ a $70 \%$, em proporção $1: 2, \mathrm{~m} / \mathrm{v}$ por 12 horas na capela de exaustão seguida de secagem por 12 horas a temperatura de $60^{\circ} \mathrm{C}$. Na carbonização, utilizouse a taxa de aquecimento de $10^{\circ} \mathrm{C} / \mathrm{min}$ até a temperatura de $450^{\circ} \mathrm{C}$, permanecendo nesta temperatura por 2 horas. Para a produção de CAG-B foram imersos 1,5 kg do material precursor em 3,0 litros de solução de $\mathrm{NaOH}$, (2,0 mols/L) na proporção massa por volume (1:2) por 24 horas capela de exaustão, para impregnação total dos caroços pelo agente ativante, logo após foi submetido a secagem em estufa por 24 horas a temperatura de $60^{\circ} \mathrm{C}$. Na carbonização, utilizou-se um forno tipo mufla com reator para a 
realização do tratamento térmico, com taxa de aquecimento de $10^{\circ} \mathrm{C} / \mathrm{min}$ até a temperatura de $400^{\circ} \mathrm{C}$, permanecendo nesta temperatura por 1 hora.

A Figura 4 mostra os carvões produzidos e ativados CAG-A (a) e CAG-B (b) antes da trituração.

Figura 4 - CAG-A (a) e CAG-A (B).
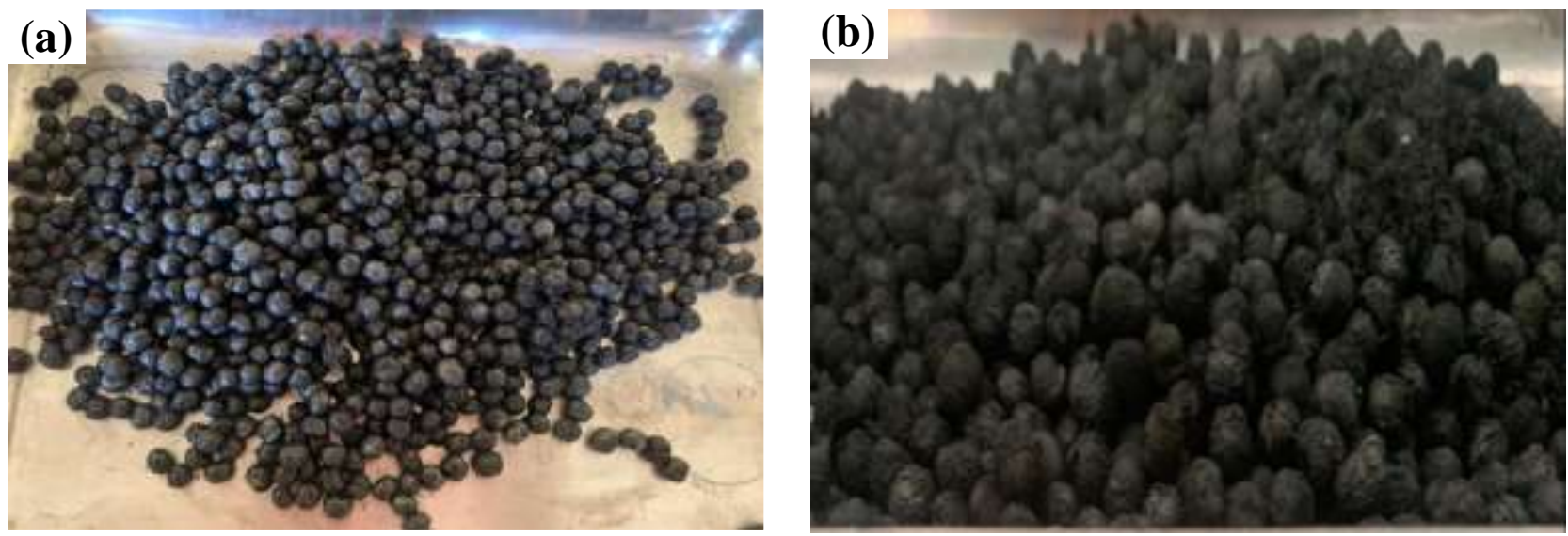

Fonte: Autores (2021).

O resfriamento de CAG-A e CAG-B mostrados na Figura 4, ocorreu naturalmente até atingir a temperatura ambiente, logo em seguida, ambos os carvões foram lavados várias vezes em água destilada, até o lixiviado atingir valores de pH próximo ao neutro, seguido de secagem em estufa por 24 horas a temperatura de $102^{\circ} \mathrm{C}( \pm 3)$. Após isso, os carvões foram triturados e peneirados sendo o material que seguiu para os estudos de adsorção foi aquele que ficou retido na peneira de 40 mesh e passante na de 35 mesh, considerado granular de acordo com a IUPAC.

\subsection{Espectroscopia no infravermelho por transformada de Fourier - FTIR}

Com intuito de verificar os grupos funcionais de superfície, os espectros de infravermelho foram obtidos em um espectrômetro Thermo, modelo Nicolet IS5, com varredura na região entre 4000 a 400cm -1. As amostras foram preparadas com pastilhas de brometo de potássio $(\mathrm{KBr})$.

\section{3 Área superficial específica}

As isotermas de adsorção e dessorção de nitrogênio foram obtidas em um equipamento Micromeritics Tristar II modelo 3020, para determinação da área superficial específica $\left(\mathrm{S}_{\mathrm{BET}}\right)$ e o tamanho médio dos poros das amostras. Antes das medidas, aproximadamente $0,1 \mathrm{mg}$ de amostra foram submetidas a um pré-tratamento sob vácuo, a $300^{\circ} \mathrm{C}$ por $3 \mathrm{~h}$. Os resultados da área superficial foram determinados pelo método padrão de Brunauer-Emmett-Teller (BET). O volume de poros e o diâmetro de poros foi obtido de acordo com o método de Barret-Joyner-Halenda (BJH).

\subsection{Análise termogravimétrica - ATG e análise térmica diferencial - ATD}

O ensaio de ATG/ATD para estudar o comportamento térmico dos CA's foi realizado em equipamento da marca Shimadzu, modelo ATG-50, seguindo as condições de análise a seguir: a amostra é pesada continuamente, em uma balança analítica sensível, acoplada ao forno, enquanto a temperatura é modificada desde a temperatura ambiente até $1000{ }^{\circ} \mathrm{C}$, a razão de aquecimento é de $20^{\circ} \mathrm{C} / \mathrm{min}$, em atmosfera de nitrogênio, operando com vazão de $50 \mathrm{~mL} / \mathrm{min}$. 


\subsection{Microscopia eletrônica de varredura - MEV e espectroscopia por energia dispersiva - EDS}

Para a análise textural, estrutural e química das amostras de CAG-A e CAG-B foi utilizada a técnica de Microscopia Eletrônica de Varredura (MEV) e Espectroscopia por Energia Dispersiva (EDS). Foi utilizado o equipamento Microscópio Eletrônico de Varredura, modelo Shimadzu (Veja 3 - sbutescan) equipado com um espectrômetro de energia dispersiva de raios X (EDS) AZTec Energy X-Act, Oxford.

\subsection{Isotermas de adsorção e efeito da concentração inicial}

O efeito da concentração inicial bem como as isotermas foram obtidas adicionando $50 \mathrm{mg}$ do CAG-A e $15 \mathrm{mg}$ do CAGB em contato com $50 \mathrm{~mL}$ de solução do corante com concentrações iniciais de 100 a $380 \mathrm{mg} / \mathrm{L}$ para o CAG-B e 60 a $380 \mathrm{mg} / \mathrm{L}$ para o CAG-A em frascos 12rlenmeyer de $250 \mathrm{~mL}$. A mistura permaneceu em agitação constante ( $90 \mathrm{rpm}$ ) sob temperatura constante de $30^{\circ}$, utilizando um Banho Maria Dubnoff com agitação e movimento recíproco durante 12 horas. Ao término do tempo necessário para o equilíbrio, as amostras foram filtradas com o auxílio de papel filtro quantitativo para assim realizar as leituras em espectrofotômetro, afim de determinar as concentrações remanescentes do corante.

Assim, a concentração restante do corante foi determinada por Espectrofotômetro UV/Vis (Agilent Technologies Cary 60), comprimento de onda $617 \mathrm{~nm}$. Com os dados obtidos, foi possível determinar a quantidade de corante adsorvida para cada concentração inicial, através da equação (1). Com isso, plotou-se o gráfico $\mathrm{q}_{\mathrm{e}}$ versus $\mathrm{C}_{\mathrm{e}}$, em seguida os dados foram ajustados aos modelos matemáticos de adsorção de Langmuir e Freundlich.

\subsection{Cinética}

No estudo cinético, foram adicionados $50 \mathrm{~mL}$ de solução preparadas com concentração de $100 \mathrm{mg} \cdot \mathrm{L}^{-1}$, a partir da diluição da solução estoque, em 12rlenmeyer de $250 \mathrm{~mL}$ contendo $50 \mathrm{mg}$ de massa do CAG-A e $20 \mathrm{mg}$ de CAG-B, logo após foram acondicionados para agitação no Banho Maria Dubnoff com temperatura constante de $30^{\circ}$ e velocidade de agitação de 90 rpm. O tempo de retirada das amostras contendo a solução foram de 5, 10, 15, 20, 30, 40, 60, 90, 120, 240, 360 e 720 minutos, e em cada intervalo a amostra foi filtrada utilizando papel filtro quantitativo seguida de leitura no espectrofotômetro UV/Vis no comprimento de onda de $617 \mathrm{~nm}$. Obtido os resultados, os dados foram ajustados aos modelos de pseudoprimeira ordem, pseudossegunda ordem e difusão intrapartícula para observar o mecanismo de adsorção e as etapas controladoras do processo.

Para os estudos de equilíbrio e cinética foram preparadas soluções de estoque com concentração de $400 \mathrm{mg} / \mathrm{L}$ do corante Verde Malaquita. Todos os ensaios foram realizados em duplicata.

\section{Resultados e Discussão}

\subsection{Espectroscopia no infravermelho por transformada de Fourier}

De acordo com Yourgun e Yildiz (2015), os carvões ativados têm uma grande variedade de grupos funcionais de superfície e que por sua vez, dependem do material precursor e do método de ativação, sendo de fundamental importância a identificação desses grupos, uma vez que são responsáveis por determinar as propriedades de superfície do carvão ativado. Os espectros de FTIR de CAG-A e CAG-B mostradas na Figura 5, mostram que diferentes grupos funcionais foram produzidos durante o processo de ativação.

O espectro FTIR mostra bandas em 2962,45 $\mathrm{cm}^{-1}$ e 2920,74 $\mathrm{cm}^{-1}$ do CAG-A e CAG-B respectivamente, que são atribuídas à vibração de alongamento alifático $\mathrm{C}-\mathrm{H}$, indicando grupos metoxibenzeno, metilo e metileno. As bandas em 1773,74 $\mathrm{cm}^{-1}$ e 1685,36 cm $\mathrm{cm}^{-1}$ em CAG-B correspondem ao alongamento $\mathrm{C}=\mathrm{O}$ do grupo acetila na hemicelulose (Tang et al., 2012) assim como também podem ser devido à vibração de deformação N - H (Shamsuddin et al, 2016). Os picos em 1570,84 cm-1 e 1430,15 
$\mathrm{cm}^{-1}$ são atribuídos a estruturas de carboxilcarbonato. Esses picos também podem ser atribuídos a grupos funcionais contendo oxigênio $\mathrm{C}$ - O de grupos carboxílicos ou vibração no plano de $\mathrm{O}-\mathrm{H}$ do grupo carboxílico.

Figura 5 - Espectro FTIR das amostras de CAG-A e CAG-B.

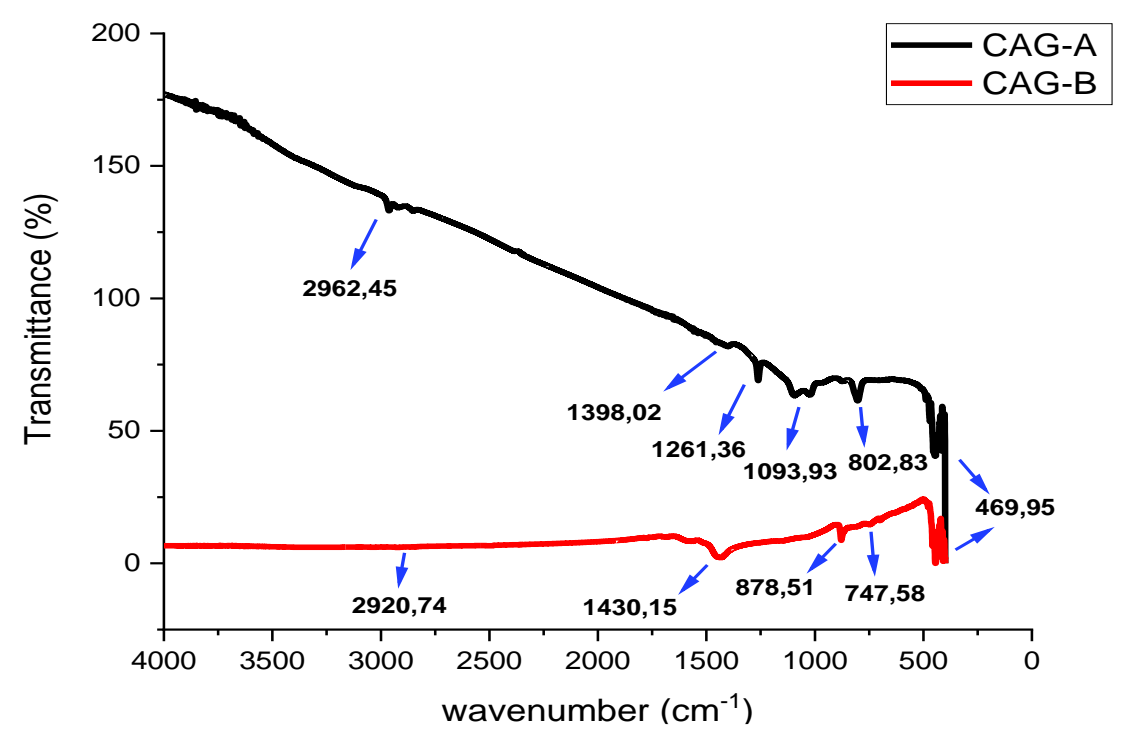

Fonte: Autores (2021).

De acordo com Fernandes et al. (2019), o pico em 878,51 $\mathrm{cm}^{-1}$ é devido ao modo de deformação fora do plano de C $\mathrm{H}$ para diferentes anéis de benzeno substituídos. As bandas em 747,58 $\mathrm{cm}^{-1}$ e $700,70 \mathrm{~cm}^{-1}$ são vibrações de torção de $\mathrm{C}-\mathrm{O}$ H. Já as bandas entre $457,84-410,00 \mathrm{~cm}^{-1}$ indicam o estiramento do grupo $\mathrm{C}-\mathrm{C}$ ou $\mathrm{C}-\mathrm{H}$ associado muito provavelmente a presença de anéis aromáticos (Clark, 2010).

A ausência dos picos $1773,74 \mathrm{~cm}^{-1}, 1685,38 \mathrm{~cm}^{-1}, 1570,64 \mathrm{~cm}^{-1}$ e 1430, $15 \mathrm{~cm}^{-1}$ em CAG-A indica decomposição de grupos funcionais presentes nos hidrocarbonetos oxigenados, refletindo a estrutura dos carboidratos da celulose e da hemicelulose (Shamsuddin et al, 2016). O pico em 1398,02 $\mathrm{cm}^{-1}$ é geralmente indica a presença de carbonos oxidados e pode ser atribuída ao alongamento C - O em ácidos, álcoois, fenóis, éteres ou grupos de ésteres, presentes na superfície do precursor, os quais fazem parte da matriz celulósica. No entanto, também é característico de fósforo e compostos fosfocarbonáceos presentes nos carvões ativados por $\mathrm{H}_{3} \mathrm{PO}_{4}$ (Xu et al., 2014; Yourgun \& Yildiz, 2015).

Ainda sobre a amostra CAG-A, a banda em $1261,36 \mathrm{~cm}^{-1}$ pode ser atribuída à vibração de alongamento de $\mathrm{P}=\mathrm{O}$ ligado por hidrogênio, além de vibrações de alongamento $\mathrm{O}-\mathrm{C}$ na ligação $\mathrm{P}-\mathrm{O}-\mathrm{C}$ e $\mathrm{P}=\mathrm{OOH}$. Os picos em $1093.93 \mathrm{~cm}^{-1} \mathrm{e} 1021,54$ $\mathrm{cm}^{-1}$ podem ser devido ao estiramento $\mathrm{P}^{+}-\mathrm{O}^{-}$em ésteres de ácido fosfato e à vibração simétrica na cadeia de polifosfato $\mathrm{P}-\mathrm{O}$ - P. Isso indica que houve decomposição de pelo menos alguns dos grupos de oxigênio e carbono na superfície do material carbonáceo, devido ao desenvolvimento de vibrações $\mathrm{C}-\mathrm{H}$ bem como também um aumento de grupos funcionais contendo fósforo (Puziy et al., 2007; Yourgun \& Yildiz, 2015).

A banda $802,83 \mathrm{~cm}^{-1}$ indica uma deformação fora do plano de $\mathrm{C}-\mathrm{H}$ para diferentes anéis benzênicos de substituição (Fernandes et al., 2019). Segundo Clark (2010) As bandas entre 488,29 - 416,80 $\mathrm{cm}^{-1}$ assim como no CAG-B, mostram o estiramento de $\mathrm{C}-\mathrm{C}$ ou $\mathrm{C}-\mathrm{H}$ sugerindo a presença de anéis aromáticos. O resultado da espectroscopia FTIR tanto para o CAGA quanto para CAG-B indica que os carvões produzidos são ricos em grupos funcionais de superfície.

Segundo Almeida (2015), esses grupos funcionais de superfície predominantemente ácido dão aos carvões ativados (CAG-A e CAG-B) propriedades iônicas, isto devido a superfície do adsorvente ficar carregada negativamente em virtude das 
características dos grupos funcionais. Isto favorece a adsorção do Verde Malaquita, por ser um corante básico, possui moléculas carregadas positivamente, o que facilita a troca catiônica com ambos os carvões.

\subsection{Análise termogravimétrica e análise térmica diferencial}

$\mathrm{O}$ comportamento térmico do carvão ativado com $\mathrm{H}_{3} \mathrm{PO}_{4}$ e $\mathrm{NaOH}$ são mostrados na figura 6 através das curvas de ATG/DrATG e ATD.

Figura 6 - Curvas ATG/ATD para CAG-A (a) e CAG-B (b).
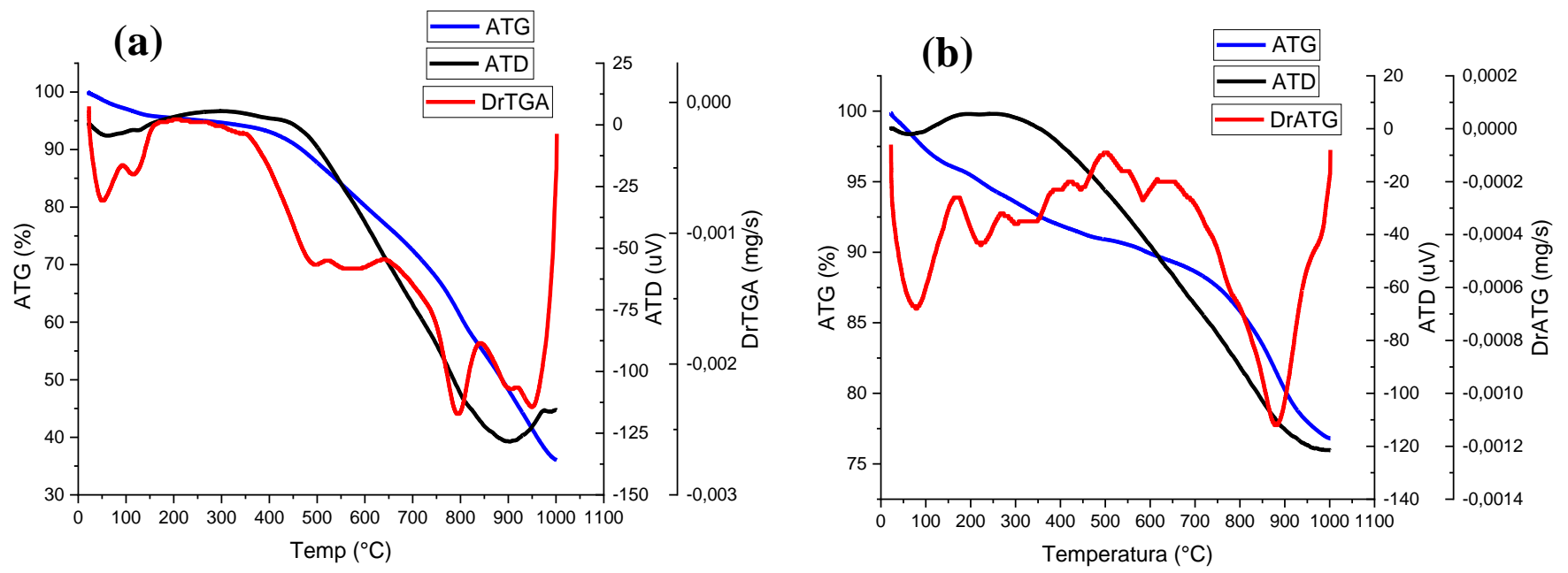

Fonte: Autores (2021).

A degradação térmica do CAG-A ocorreu em quatro etapas principais. As duas primeiras etapas acontecem até $246^{\circ} \mathrm{C}$, apresentando dos picos endotérmicos, o primeiro em torno de $72{ }^{\circ} \mathrm{C}$ (cerca de $4 \%$ ), relacionados à perda de umidade e o segundo pico em $213^{\circ} \mathrm{C}$ ( $2 \%$ de perda de massa) que pode ser atribuído aos componentes voláteis e gases leves, como $\mathrm{CO}_{\text {e }} \mathrm{CO}_{2}$, resultado da quebra térmica de ligações fracas na estrutura polimérica dos principais componentes da biomassa e da formação de ligações mais fortes e estáveis. A terceira etapa correspondente a perda de massa de 4,5\% ocasionada pela reação do $\mathrm{H}_{3} \mathrm{PO}_{4}$ com biopolímero para formação de micro e mesoporos. A quarta perda de massa $(12,75 \%)$ em $852^{\circ} \mathrm{C}$, pode ser atribuída à oxidação final do material de carbono após a reação com $\mathrm{H}_{3} \mathrm{PO}_{4}$, devido a decomposição das pontes de fosfato (De Souza et al., 2018 ; Xu et al., 2014).

Para o CAG-B, pode-se verificar que as perdas de massas ocorrem em quatro etapas distintas. De $34^{\circ} \mathrm{C}$ a $79^{\circ} \mathrm{C}$ ocorre a primeira perda de massa na ordem de $2,4 \%$, referente a perda de umidade adquirida do armazenamento, depois observa-se entre $95^{\circ} \mathrm{C}$ a $150{ }^{\circ} \mathrm{C}$ uma leve perda de aproximadamente $2 \%$ devido a decomposição dos resquícios da matéria orgânica. A oxidação do material carbonáceo ocorreu em duas etapas, iniciando em $505^{\circ} \mathrm{C}$ com perda de massa de $18 \%$ (ATG), em seguida uma perda de massa em $750^{\circ} \mathrm{C}$ (no total de $1,132 \mathrm{mg}$ ) sendo $21 \%$ da massa conforme curva ATG (Xu et al., 2014).

O gráfico de TGA para o CAG-A mostra que a temperatura de ativação a $450{ }^{\circ} \mathrm{C}$ corresponde a uma queima de massa de 39,6\%, que pode ser atribuída essencialmente à perda de massa relacionados a formação de micro e mesoporos devido à reação de ativação com $\mathrm{H}_{3} \mathrm{PO}_{4}$. Isso significa que menos de 76,4\% do precursor do caroço de açaí é queimado pela reação de ativação. Resultados parecidos foram encontrados por Benadjemia et al. (2011) e Xu et al. (2014) utilizando como material precursor folhas de alcachofra e capim-bravo respectivamente. 


\section{3 Área superficial específica}

A Figura 7 mostra os resultados das isotermas de adsorção de $\mathrm{N}_{2}$ (Análise BET) para CAG-A (a) e CAG-B (b). O CAGA apresenta característica das isotermas de adsorção do tipo I, que segundo Mangueira (2014) está relacionada com a adsorção em pequenas superfícies externas de materiais microporosos, dando forma a um processo adsortivo em monocamada e também exibe um comportamento característico do tipo IV, indicando estrutura mesoporosa do carvão, através da presença da histeres. No entanto, essa histerese apresentada na figura 7a (tipo H3), indica que a contribuição de volume de mesoporos é pequena em relação à microporosidade, que é predominante. (Guimarães, 2006; Andrade, 2014).

Figura 7 - Isotermas de $\mathrm{N}_{2}$ para CAG-A (a) e CAG-B (b).
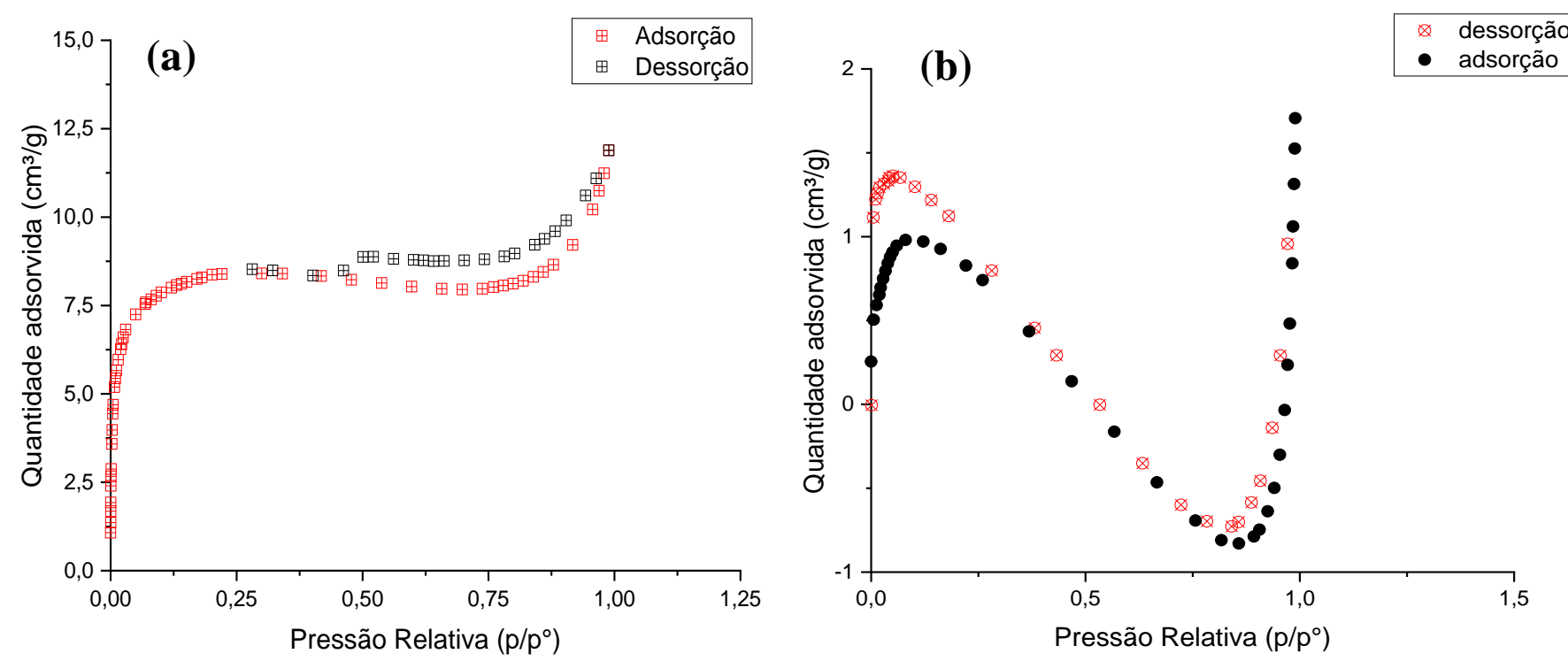

Fonte: Autores (2021).

Já o CAG-B (representado na Figura 7b) segundo a classificação da IUPAC (Fan et al., 2013) tende para um misto entre as isotermas do tipo I e II, com predominância do tipo II. Segundo Reis (2013) e Almeida (2015) em relação ao tipo I, indica que o carvão possui elevado volume de microporos sugerindo que nos instantes iniciais o processo adsortivo limitou-se a uma camada. No que concerne ao tipo II, sugere uma adsorção em multicamadas, ou seja, o ponto de inflexão da curva mostra que a adsorção em monocamada está completa, iniciando o processo de em múltiplas camadas em função do aumento da temperatura, até atingir a saturação do adsorvente. O CAG-B apresentou uma adsorção negativa, ou seja, uma perda de soluto (entre as pressões relativas de 0,5 e $1,0 \mathrm{p} / \mathrm{p}^{\circ}$ ), que pode estar relacionada a um desprendimento da quantidade inicial do soluto dos sítios ativos do carvão.

Os valores de $\mathrm{S}_{\mathrm{BET}}$ obtidos neste estudo foram de $2,8 \mathrm{~m}^{2} / \mathrm{g}$ de área superficial, volume de poros de $0,001642 \mathrm{~cm}^{3} / \mathrm{g}$ e tamanho médio dos poros 24,8 Å para o CAG-B e $29,1 \mathrm{~m}^{2} / \mathrm{g}$ de área superficial, um volume de $0,01838 \mathrm{~cm}^{3} / \mathrm{g}$ e tamanho médio dos poros de 25,8 $\AA$. A baixa ASE desse estudo está relacionada com a limitação do equipamento que não consegue analisar amostras microporosas, dando um resultado muito aquém do real. Shultz (2012) também não conseguiu observar microporosidade do material porque esta análise estava fora do limite de detecção, sendo esta determinada indiretamente por outra análise.

Vários estudos utilizando biomassa como carvão ativado para remoção do Verde Malaquita que apresentaram capacidade de adsorção inferior a capacidade mostrada neste estudo, tiveram áreas específicas bem mais alta. Por exemplo, Zhang et al. (2008) ao utilizar a raiz da Cana de açúcar obteve 158 m²/g, e Yu et al. (2017) utilizando a biomassa de Taboa, 
obteve $441,12 \mathrm{~m}^{2} / \mathrm{g}$ de área superficial. No entanto ambos os trabalhos apresentaram menor capacidade de adsorção (210,18 $\mathrm{m}^{2} / \mathrm{g}$ e $8,69 \mathrm{~m}^{2} / \mathrm{g}$ respectivamente).

\subsection{Microscopia eletrônica de varredura e espectroscopia por energia dispersiva}

As Figuras $8 \mathrm{a}$ e $8 \mathrm{~b}$ a seguir mostram as micrografias das amostras do CAG-A e CAG-B respectivamente em 100x e as Ffiguras $8 \mathrm{c}$ e $8 \mathrm{~d}$ em $1 \mathrm{k}$.

Figura 8 - Micrografia para CAG-A (a) e CAG-B (b) em 100x e CAG-A (c) e CAG-B (d) em 1k.
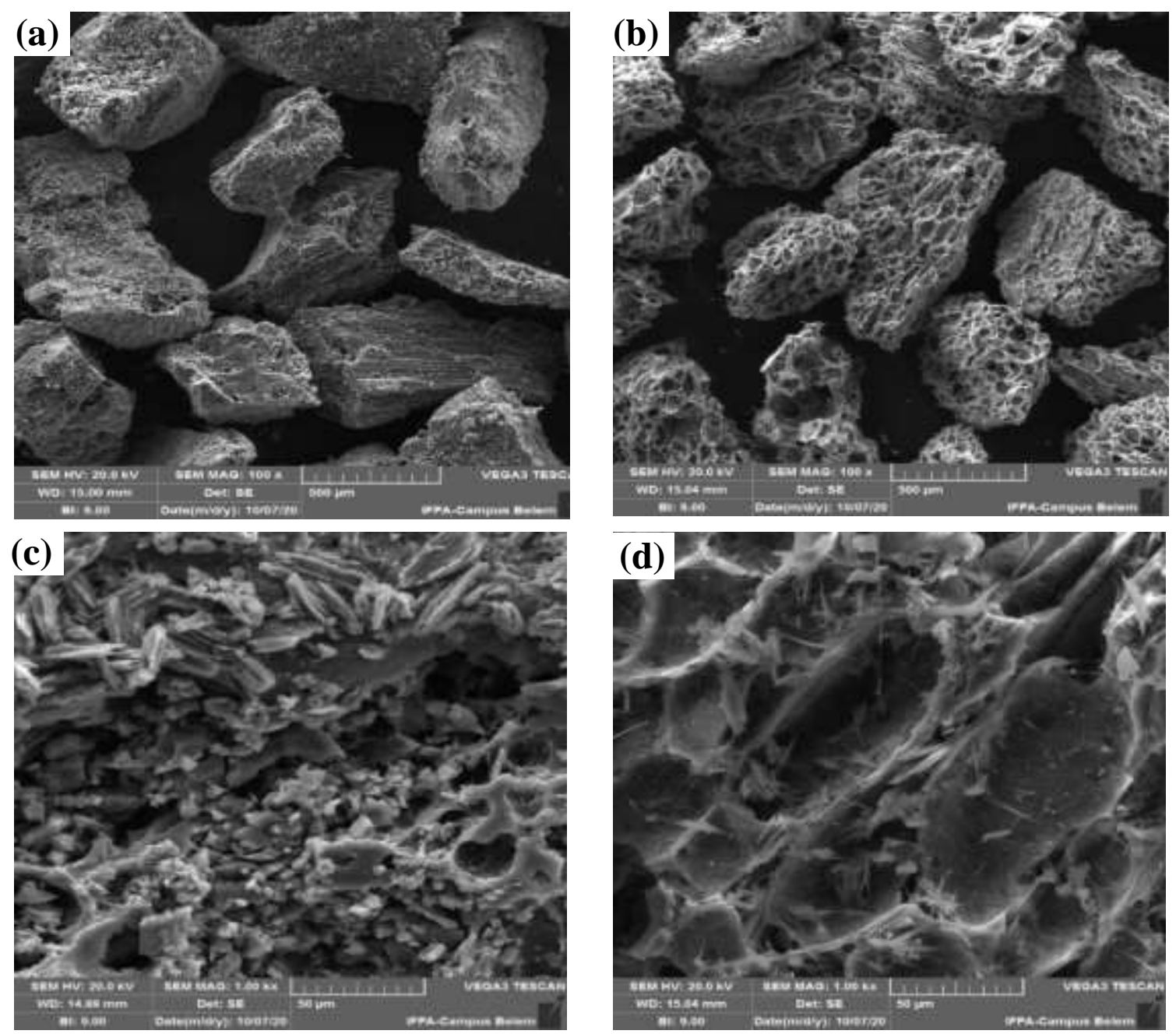

Fonte: Autores (2021).

Ao analisar as micrografias da amostra CAG-A (Figura 8a e 8c), observa-se uma superfície com cavidades irregulares com partes lisas e heterogênias, o que significa que algumas partes não foram totalmente desobstruídas apresentando uma desvantagem em relação a amostra anterior, mas ainda assim teve uma porosidade bem desenvolvida assim como a amostra de carvão realizada no MEV de Kaouah, et al. (2013) que atesta mudanças substanciais provocadas pelo $\mathrm{H}_{3} \mathrm{PO}_{4} \mathrm{e}$ uma superfície adsorvente com caráter microporoso predominante, a qual influencia na área superficial.

A micrografia para CAG-B (100x) mostra que após o processo de pirólise e ativação houve uma modificação na estrutura do precursor, com formação de poros em forma de crateras uniformes. Na imagem de $1 \mathrm{k}$ da mesma, nota-se que as cavidades dos poros são alongadas e com aberturas arredondadas, esta microestrutura homogênea pode ser favorável para a adsorção do corante. 
A análise elementar do CAG-A e CAG-B são mostradas na Tabela 1. Os resultados das amostras apresentam em comum o carbono (C) com o maior teor na matriz (26,3\% e 29,91\%), em seguido o oxigênio (O) com 8,64\% no CAG-B e $16,4 \%$ no CAG-A e para o potássio com uma das menores concentrações, 0,6\% para o CAG-B e 2,89\% para o CAG-A. Os resultados do CAG-B indicaram o sódio $(\mathrm{Na})$ com uma concentração ainda considerável $(5,44 \%)$, e o cloro $(\mathrm{Cl})$ em quantidade irrelevante $(0,1 \%)$. Diferentemente do CAG-B, o resultado de CAG-A detectou o elemento fósforo (P) com 14,21\% e constou baixa concentração para o cálcio $(\mathrm{Ca})$ com apenas $0,26 \%$.

Tabela 1 - Resultados do EDS para CAG-A e CAG-B.

\begin{tabular}{c|c|c}
\hline \multirow{2}{*}{ Elemento químico } & \multicolumn{2}{|c}{ Composição \% } \\
\cline { 2 - 3 } & CAG-A & CAG-B \\
\hline $\mathrm{C}$ & 29,91 & 26,43 \\
\hline $\mathrm{O}$ & 16,4 & 8,64 \\
\hline $\mathrm{P}$ & 14,21 & - \\
\hline $\mathrm{Na}$ & - & 5,44 \\
\hline $\mathrm{K}$ & 2,89 & 0,6 \\
\hline $\mathrm{Ca}$ & 0,26 & - \\
\hline $\mathrm{Cl}$ & - & 0,1 \\
\hline
\end{tabular}

Fonte: Autores (2021).

Os resultados das amostras apresentam em comum o carbono (C) com o maior teor na matriz (26,3\% e $29,91 \%)$, em seguido o oxigênio $(\mathrm{O})$ com $8,64 \%$ no CAG-B e 16,4\% no CAG-A e para o potássio com uma das menores concentrações, $0,6 \%$ para o CAG-B e 2,89\% para o CAG-A. Os resultados do CAG-B indicaram o sódio (Na) com uma concentração ainda considerável $(5,44 \%)$, e o cloro $(\mathrm{Cl})$ em quantidade irrelevante $(0,1 \%)$. Diferentemente do CAG-B, o resultado de CAG-A detectou o elemento fósforo $(\mathrm{P})$ com $14,21 \%$ e constou baixa concentração para o cálcio (Ca) com apenas $0,26 \%$.

Esta diferença ocorre devido aos diferentes agentes de ativações utilizados para cada amostra. O carbono presente nas duas amostras é o componente proeminente que indica a natureza carbonácea que, por sua vez, confere porosidade ao CA. O oxigênio em concentrações elevadas nos dois resultados influencia fortemente na capacidade de adsorção do CA pois, segundo Guimarães (2006) quanto maior o conteúdo de oxigênio mais ácida é a dispersão aquosa do CA, e isto confere propriedades de trocas catiônicas.

A imagens em camadas da análise EDS (Figura 9) mostra a distribuição dos elementos na superfície da amostra. 
Figura 9 - Imagens em camadas das amostras CAG-A (a) e CAG-B (b).
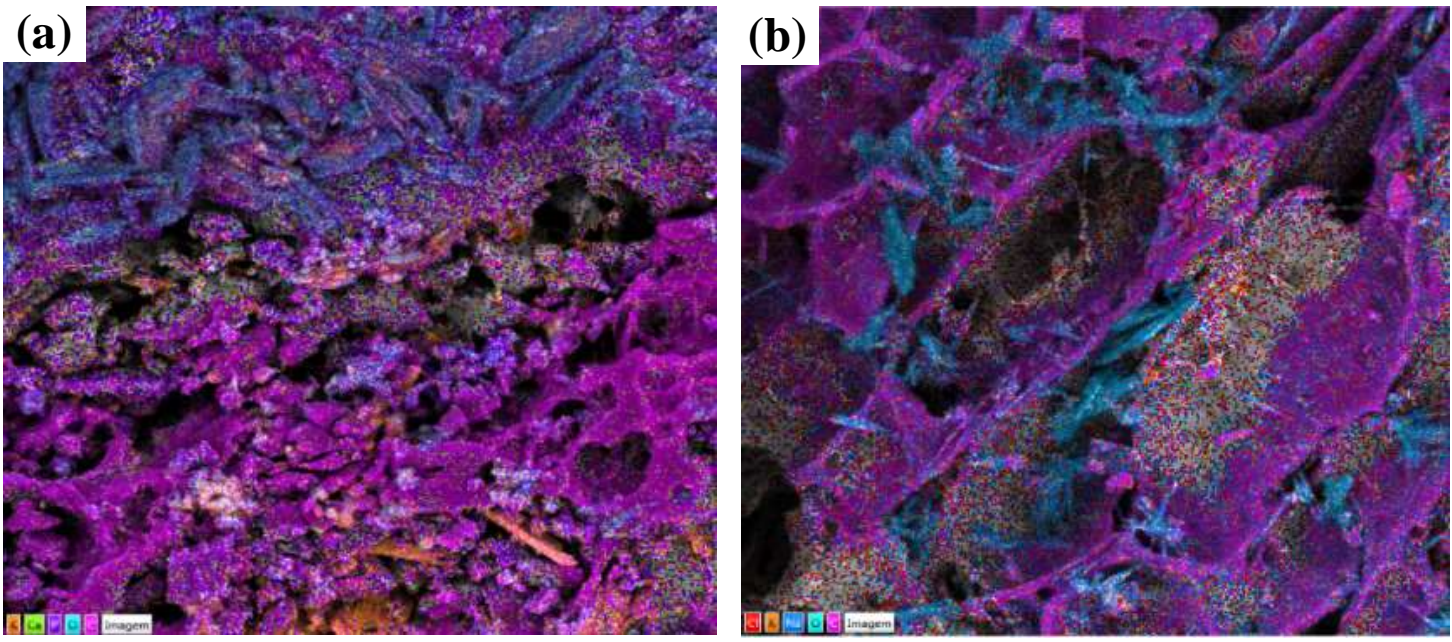

Fonte: Autores (2021).

A cor lilás representando o carbono destaca-se em toda a superfície comprovando que este elemento está de fato em maior porcentagem como mostra as tabelas anteriores. É possível visualizar também o teor expressivo de oxigênio, na figura $9 \mathrm{~b}$ está aglomerado com o sódio representado pelas cores azul e verde, e na Figura 9a o oxigênio aparece juntamente com o fósforo, nas cores verde e roxo.

\subsection{Isotermas de adsorção}

As isotermas de adsorção são comumente usadas para avaliar a relação entre a concentração de adsorbato e a capacidade de adsorção do adsorvente. Assim, os dados de equilíbrio para o CAG-A e CAG-B ajustados às isotermas de Langmuir e Freundlich, são mostrados nas Figuras 10a e 10b para CAG-A e 11a, 11b para CAG-B.

Figura 10 - Ajuste do CAG-A aos modelos de Langmuir (a) e Freundlich (b).
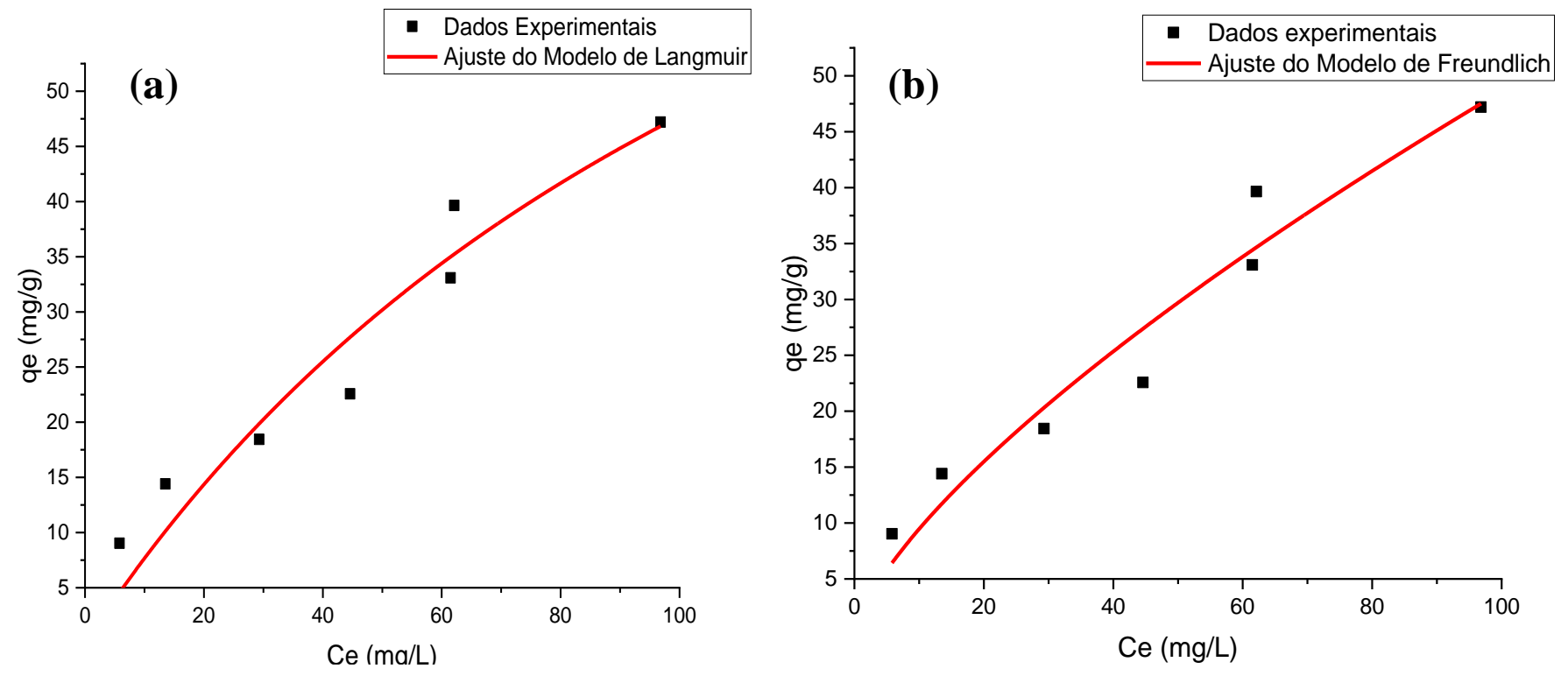

Fonte: Autores (2021). 
Figura 11 - Ajuste do CAG-B aos modelos de Langmuir (a) e Freundlich (b).
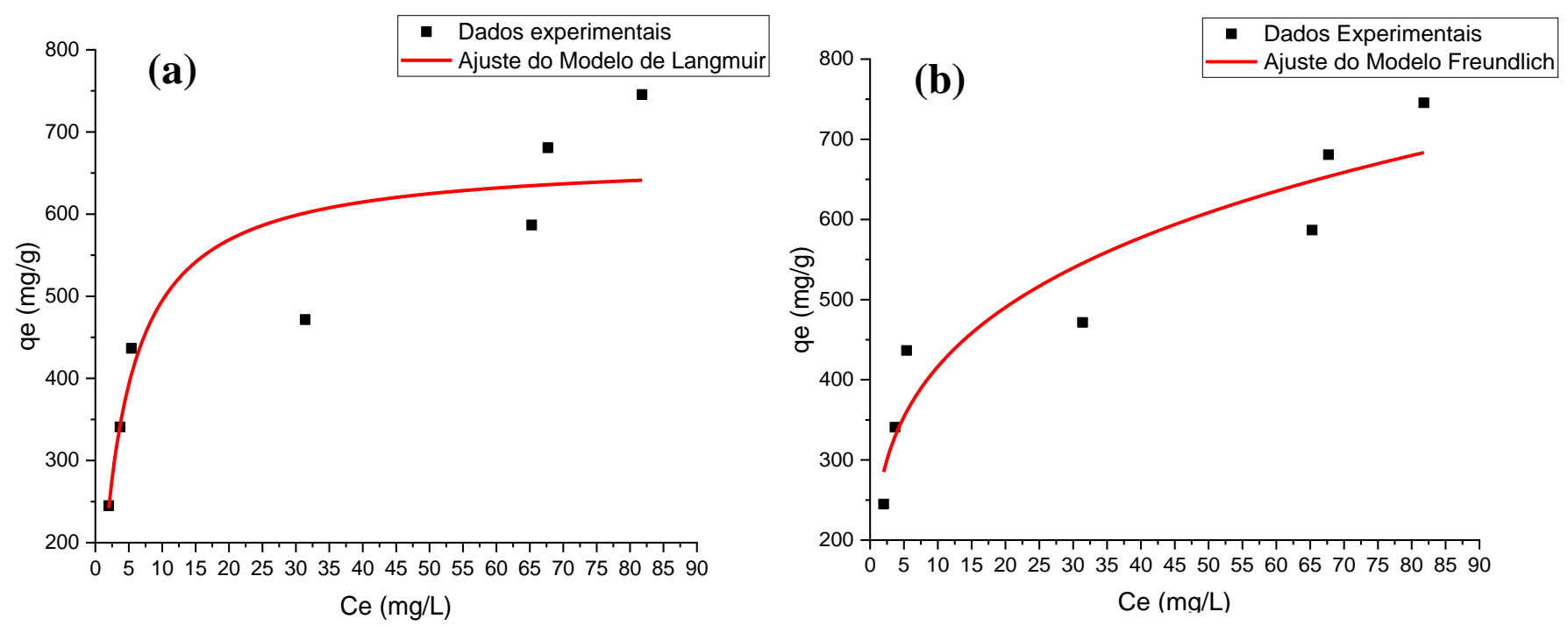

Fonte: Autores (2021).

As Figuras 10a, 11a e 11b mostram isotermas do tipo L (Figura 10a do tipo $\mathrm{L}_{2}$ e Figuras 11a e 11b são do tipo $\mathrm{L}_{1}$ ) sugerindo que, segundo Oliveira (2013) possuem alta afinidade entre adsorvente - adsorbato em baixas concentrações, ou seja, a medida que aumenta a concentração da solução, diminui a disponibilidade dos sítios de adsorção. Já na Figura 10b a isoterma é do tipo $\mathrm{H}$, caracterizando uma superfície com alta afinidade pelo soluto com uma forte interação eletrostática entre adsorvente e adsorbato, isto é, a remoção do soluto no início é muito alta e logo após o equilíbrio é atingido (Porpino, 2009). Essa interação forte, segundo Azevedo (2015), está relacionada à interação molecular das cargas negativas presentes nos grupos funcionais de superfície do adsorvente e o caráter básico do corante Verde Malaquita (catiônico).

Na Tabela 2 são apresentados os valores dos parâmetros dos modelos de isoterma de Langmuir e Freundlich para a adsorção do Verde Malaquita nos carvões ativados com $\mathrm{NaOH}$ e $\mathrm{H}_{3} \mathrm{PO}_{4}$.

Tabela 2 - Valores dos parâmetros para equilíbrio segundo os modelos de Langmuir e Freundlich.

\begin{tabular}{c|c|c|c}
\hline \multirow{2}{*}{$\begin{array}{c}\text { MODELO DE } \\
\text { ISOTERMA }\end{array}$} & \multirow{2}{*}{ PARÂMETRO } & \multicolumn{2}{c}{ ADSORÇÃO DO VERDE MALAQUITA } \\
\cline { 2 - 4 } & & \multicolumn{1}{c}{ CAG-A } & CAG-B \\
\cline { 2 - 4 } & $\mathrm{Q}_{\mathrm{max}}(\mathrm{mg} / \mathrm{g})$ & 113,9 & 668,88 \\
\cline { 2 - 4 } & $\mathrm{K}_{\mathrm{L}}(\mathrm{L} / \mathrm{mg})$ & 0,0072 & 0,28357 \\
\cline { 2 - 4 } & $\mathrm{R}_{\mathrm{adj}}{ }^{2}$ & 0,90 & 0,80 \\
\cline { 2 - 4 } & $\mathrm{SQR}$ & 90,36 & 33071,83 \\
\hline \multirow{5}{*}{ Freundlich } & $\mathrm{N}$ & 4,2 & 241,97 \\
\cline { 2 - 4 } & $\mathrm{K}_{\mathrm{f}}\left(\mathrm{mg} / \mathrm{g}(\mathrm{L} / \mathrm{mg})^{1 / n}\right)$ & 1,84 & 0,87 \\
\cline { 2 - 4 } & $\mathrm{R}_{\mathrm{adj}}{ }^{2}$ & 0,99 & 21361,55 \\
\cline { 2 - 4 } & $\mathrm{SQR}^{*}$ & 55,12 & \\
\hline
\end{tabular}

Fonte: Autores (2021).

Conforme mostra a Tabela 2 o modelo de equilíbrio que se ajustou satisfatoriamente aos dados experimentais para o CAG-A, foi o modelo de Freundlich, apresentando $\mathrm{R}_{\mathrm{adj}}{ }^{2}=0,99$ e valor de $\mathrm{n}$ consideravelmente maior que a unidade $(\mathrm{n}=4,2)$. Essa representação satisfatória dos dados de isoterma para este modelo, indica segundo Marrakchi et al. (2016), que a adsorção do Verde Malaquita ocorreu em múltiplas camadas na superfície heterogênea do CAG-A.

Assim como para o CAG-A, para o carvão ativado com $\mathrm{NaOH}$, o modelo matemático que melhor se ajustou aos dados experimentais foi o modelo de Freundlich, sugerindo que a adsorção ocorreu em multicamadas heterogêneas, de acordo com o 
valor de $\mathrm{R}_{\mathrm{adj}}{ }^{2}=0,87$. Além disso, segundo Islam et al. (2017) o parâmetro empírico $\mathrm{n}$ mede a extensão da favorabilidade do processo de adsorção, onde a adsorção favorável pode ser deduzida do valor de n que excede a unidade. Neste estudo, o valor de n é 1,4 indicando assim uma adsorção favorável para o CAG-B. A comparação entre as Soma dos Quadrados dos Resíduos ( $\mathrm{SQR}$ ) de cada modelo também foi levada em consideração neste estudo, de modo que, quando ocorre maiores valores de $\mathrm{R}_{\text {adj }}{ }^{2}$ combinado com valores mais baixos de SQR, melhor é o ajuste do modelo aos dados experimentais (Azevedo, 2015). Logo, o fato de valor de SQR de Freundlich ser bem inferior ao de Langmuir comprova ser o melhor ajuste para o equilíbrio.

Outros estudos de adsorção do Verde Malaquita como Mohammad et al. (2018), Murthy et al. (2019), Datta et al. (2016), Marco (2015) dentre outras literaturas também obtiveram valores de n maior que uma unidade, demonstrando uma adsorção favorável. Além disso, os valores de $\mathrm{K}_{\mathrm{f}}$ variaram significativamente em virtude das condições de cada processo de adsorção, no caso deste experimento o $\mathrm{K}_{\mathrm{f}}$ para o CAG-A teve valor de 1,84 L/mg, enquanto que o CAG-B apresentou valor de 241,97 L/mg.

A capacidade máxima de adsorção ( $\mathrm{q}_{\max }$ ) do CAG-B foi consideravelmente alta com valor de $\mathrm{q}_{\max }=668,88 \mathrm{mg} / \mathrm{g}$ e relativamente alta para o CAG-A com valor de $\mathrm{q}_{\max }=113,9 \mathrm{mg} / \mathrm{g}$. Cao et al. (2014) obteve um valor de $\mathrm{q}_{\max }=568,10 \mathrm{mg} / \mathrm{g}$ utilizando a casca da nogueira, como matéria prima do carvão ativado, com objetivo de remover corante vermelho brilhante, Calvete et al. (2010) obteve valor de qmax $=314 \mathrm{mg} / \mathrm{g}$ na adsorção do corante laranja 16 utilizando carvão ativado da casca do fruto do pinheiro, Islam et al. (2017) obteve $359 \mathrm{mg} / \mathrm{g}$ usando carvão ativado de rattan para adsorver o azul de metileno. Notase que a capacidade de remoção de corantes do CAG-B foi superior ao dos outros autores mencionados, evidenciando uma eficiência de tratamento superior.

Uma constatação que evidencia a excelente capacidade de adsorção está relacionada aos grupos funcionais presentes na superfície do CAG-A e principalmente no CAG-B. Esse fator corrobora com os resultados obtidos no FTIR e análise elementar (EDS), uma vez que as superfícies de ambos os carvões ativados foram predominantemente de caráter ácido, caracterizado principalmente pela presença de grupos carboxílicos, ésteres, fenóis, éter, acetila e grupos contendo fósforo como por exemplo o ácido fosfato.

\subsection{Cinética de adsorção}

Para conhecer o mecanismo de adsorção bem como as etapas limitantes do processo foram ajustados aos dados experimentais da adsorção do Verde Malaquita em CAG-A e CAG-B, os modelos cinéticos de Pseudo Primeira Ordem, Pseudo Segunda Orgem e Difusão Intrapartícula. As Figuras 12a, 12b e 13 mostram os ajustes aos modelos cinéticos. 
Figura 12 - Modelos cinéticos de PPO e PSO para adsorção do Verde Malaquita em CAG-A (a) e CAG-B (b).
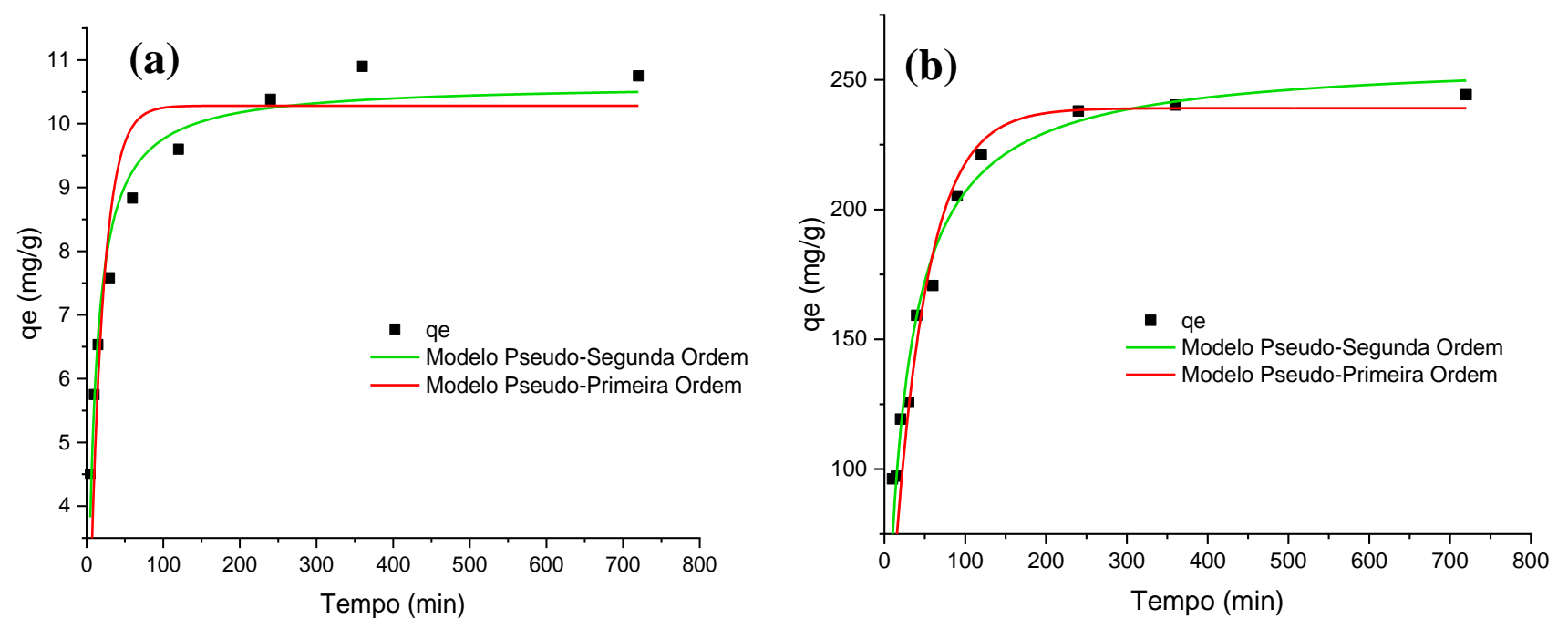

Fonte: Autores (2021).

Figura 13 - Modelo cinético de DI para adsorção do Verde Malaquita para CAG-A (a) e CAG-B (b).
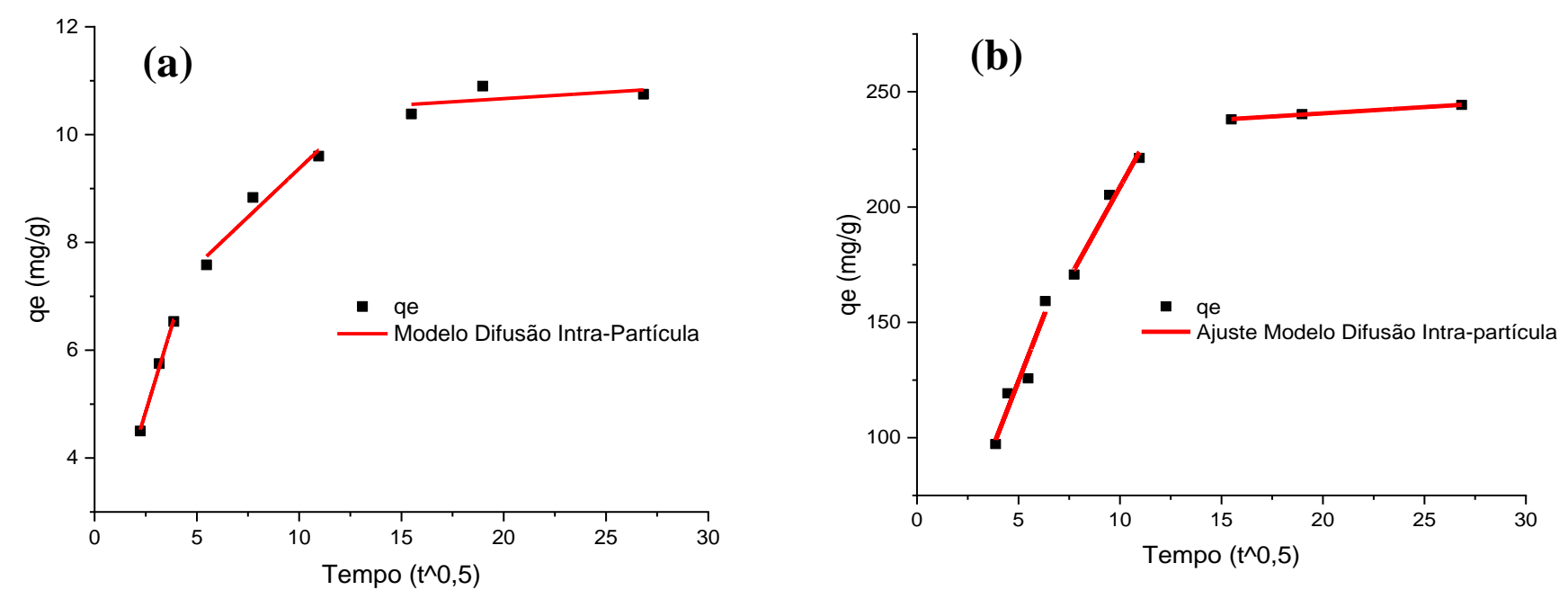

Fonte: Autores (2021).

De acordo com os resultados mostrados na Tabela 3, verifica-se que quanto aos dados experimentais do CAG-B para adsorção do Verde Malaquita, considerando o coeficiente de correlação $\mathrm{R}_{\text {adj }}{ }^{2}$ houve um ajuste satisfatório aos modelos de PPO $\left(\mathrm{R}_{\mathrm{adj}}{ }^{2}=0,99917\right)$ e PSO ( $\left.\mathrm{R}_{\mathrm{adj}}{ }^{2}=0,99948\right)$, além de um bom ajuste ao modelo de DI $\left(\mathrm{R}_{\mathrm{adj}}{ }^{2}=0,94967\right)$. No entanto, considerando outros parâmetros como $\mathrm{SQR}$ dos três modelos cinéticos aliado ao $\mathrm{R}_{\mathrm{adj}}{ }^{2}$, pode-se afirmar que o modelo PSO teve melhor ajuste dentre os demais.

O fato da adsorção do Verde Malaquita em CAG-B ter se ajustado satisfatoriamente ao modelo de pseudo primeira ordem sugere que parte do processo foi controlado por difusão através da camada controladora ao redor do adsorvente (Marco, 2015), por outro lado, o fato da PPO não ser o modelo que melhor se ajustou aos dados experimentais desse processo, pode ser atribuído a características relacionadas ao tempo de alcance do equilíbrio, pois segundo Azevedo (2015), esse modelo é representado satisfatoriamente em tempos acima de 30 minutos, uma vez que esse estudo contempla tempos abaixo de 30 minutos. 
Tabela 3 - Valores dos parâmetros para cinética segundo os modelos de PPO, PSO e DI.

\begin{tabular}{c|c|c|c}
\hline \multirow{2}{*}{$\begin{array}{c}\text { MODELOS } \\
\text { CINÉTICOS }\end{array}$} & \multirow{2}{*}{ PARÂMETROS } & \multicolumn{2}{|c}{ ADSORÇÃO DO VERDE MALAQUITA } \\
\cline { 2 - 4 } & $q_{e}(\mathrm{mg} / \mathrm{g})$ & CAG-A & CAG-B \\
\hline \multirow{3}{*}{$\begin{array}{c}\text { Pseudo Primeira } \\
\text { Ordem }\end{array}$} & $k_{l}\left(\mathrm{~min}^{-1}\right)$ & 9,94 & 239,05 \\
\cline { 2 - 4 } & $\mathrm{R}_{\mathrm{adj}}{ }^{2}$ & 0,0778 & 0,0242 \\
\cline { 2 - 4 } & $\mathrm{SQR}$ & 0,82255 & 0.99917 \\
\hline \multirow{3}{*}{$\begin{array}{c}\text { Pseudo Segunda } \\
\text { Ordem }\end{array}$} & $q_{e}(\mathrm{mg} / \mathrm{g})$ & 6,77 & 374,86 \\
\cline { 2 - 4 } & $k_{2}(\mathrm{mg} / \mathrm{g} \cdot \mathrm{min})$ & 10,62 & 258,38 \\
\cline { 2 - 4 } & $\mathrm{R}_{\mathrm{adj}}{ }^{2}$ & 0,01058 & $0,55 \times 10^{-4}$ \\
\cline { 2 - 4 } & $\mathrm{SQR}$ & 0,96088 & 234,49 \\
\hline \multirow{4}{*}{ Difusão Intrapartícula } & $C(\mathrm{mg} / \mathrm{g})$ & 1,49 & 50,02 \\
\cline { 2 - 4 } & $k_{t}\left(\mathrm{mg} / \mathrm{g} \cdot \mathrm{min}^{1 / 2}\right)$ & 0,77 & 15,86 \\
\cline { 2 - 4 } & $\mathrm{R}_{\mathrm{adj}}{ }^{2}$ & 0,3596 & 0,94967 \\
\cline { 2 - 4 } & $\mathrm{SQR}$ & 0,11 & 33,52 \\
\hline
\end{tabular}

Fonte: Autores (2021).

Outro fator importante foi o valor de $k_{l}$, indicando que o equilíbrio não foi alcançado rapidamente, apresentando valores de $k_{l}=0,0246 \mathrm{~min}^{-1}$. Segundo Marco (2015), constante $k_{l}$ descreve a velocidade com que o equilíbrio é atingido, ou seja, quanto maiores forem os valores de $k_{l}$, mais rapidamente será alcançado o equilíbrio. Yu et al. (2017) utilizou carvão ativado da biomassa de Taboa para adsorver Verde Malaquita e encontrou $k_{l}$ similar ao deste estudo $\left(k_{l}=0,0993 \mathrm{~min}^{-1}\right)$.

O ajuste satisfatório ao modelo de PSO $\left(\mathrm{R}_{\mathrm{adj}}{ }^{2}=0,99948\right.$ e baixo $\mathrm{SQR}$ comparado ao $\left.\mathrm{PPO}\right)$ revela que a etapa que controla a cinética total do processo adsortivo do Verde Malaquita pelo CAG-B é a taxa de interações eletrostáticas existentes nos sítios ativos entre adsorvente-adsorvato. Esses resultados corroboram com a análise e classificação das isotermas discutidas no tópico anterior para o equilíbrio de adsorção. Essa forte interação química apresentada pela PSO entre as moléculas do soluto e os sítios ativos da superfície do carvão ativado comprovam os resultados obtidos na caracterização de FTIR e ATG deste estudo, indicando caráter ácido do adsorvente.

Os modelos cinéticos de pseudo primeira ordem e a pseudo segunda ordem, segundo Murthy et al. (2019) não são suficientes para identificar o mecanismo de difusão. Com isto este estudo também ajustou os dados experimentais ao modelo de Difusão Intrapartícula, obtendo um bom ajuste aos dados do processo. Embora o coeficiente de correlação para DI seja $\mathrm{R}_{\mathrm{adj}}{ }^{2}=$ 0,94967 como mostra a Tabela 3, o fato da curva não passar pela origem indica que a DI não foi a etapa limitante na etapa inicial do processo (Murthy et al., 2019).

No que tange aos resultados para adsorção do Verde Malaquita com o CAG-A, verificou-se que os modelos PPO e DI não se ajustaram aos dados experimentais, observando principalmente os baixos valores de $\mathrm{R}_{\mathrm{adj}}{ }^{2}$ (menores que 0,90 ), $\mathrm{o}$ valor de SQR $(6,77)$ de PPO que é bem maior que o valor de SQR $(1,49)$ para o PSO, além disso, a capacidade de adsorção de PSO $(10,62$ $\mathrm{mg} / \mathrm{g})$ foi quase o dobro em relação ao modelo DI $(5,77 \mathrm{mg} / \mathrm{g})$.

Desta forma, o modelo PSO é o que melhor descreve o processo de adsorção entre Verde Malaquita e o CAG-A. O que implica dizer que o mecanismo de adsorção é descrito como quimissorção, envolvendo forças de valência por meio do compartilhamento ou troca de elétrons entre o adsorvente e o adsorbato como forças covalentes (Bulut, 2008), isto é, o Verde Malaquita (que é um corante básico, por tanto catiônico) com carga oposta ao CAG-A (carregado negativamente) favorece o processo de adsorção. Além disso, considerando o valor de $k_{2}=0,01052 \mathrm{mg} / \mathrm{g}$.min, denota-se que o processo de adsorção é lento, e o equilíbrio não é atingido de forma rápida, pois segundo Marco (2015), a constante $k_{2}$ também indica um fator de escala temporal, que quanto maior for o valor dessa constante, mais rápido será atingido o equilíbrio.

Resultados análogos foram encontrados em estudos envolvendo a adsorção do Verde Malaquita. Datta et al. (2016) utilizou um composto ferromagnético, Azaman et al. (2018) utilizando carvão ativado da casca de coco, Santhis et al. (2010) 
utilizou o epicarpo de Ricinus Communis e Mohammad et al. (2018) utilizando carvão ativado do casco de sementes entre outros estudos. Em todos esses estudos foram obtidos parâmetros muito semelhantes ao desta pesquisa.

\subsection{Efeito da concentração inicial do adsorbato}

A Figura 14 mostra os resultados para o efeito da concentração inicial do Verde Malaquita para o equilíbrio em CAGA (a) e CAG-B (b). A remoção do Verde Malaquita para o CAG-A, alcançou valor máximo de adsorção em torno de 91,5\% (Figura 14a) diminuindo a porcentagem de remoção para aproximadamente 78,5\% nas três primeiras concentrações, enquanto que pra CAG-B (Figura 14b), o valor máximo atingido foi de aproximadamente 98\%, e diminui lentamente para as duas próximas concentrações em $97 \%$ e $96,8 \%$.

Figura 14 - Efeito da concentração inicial para CAG-A (a) e CAG-B (b).
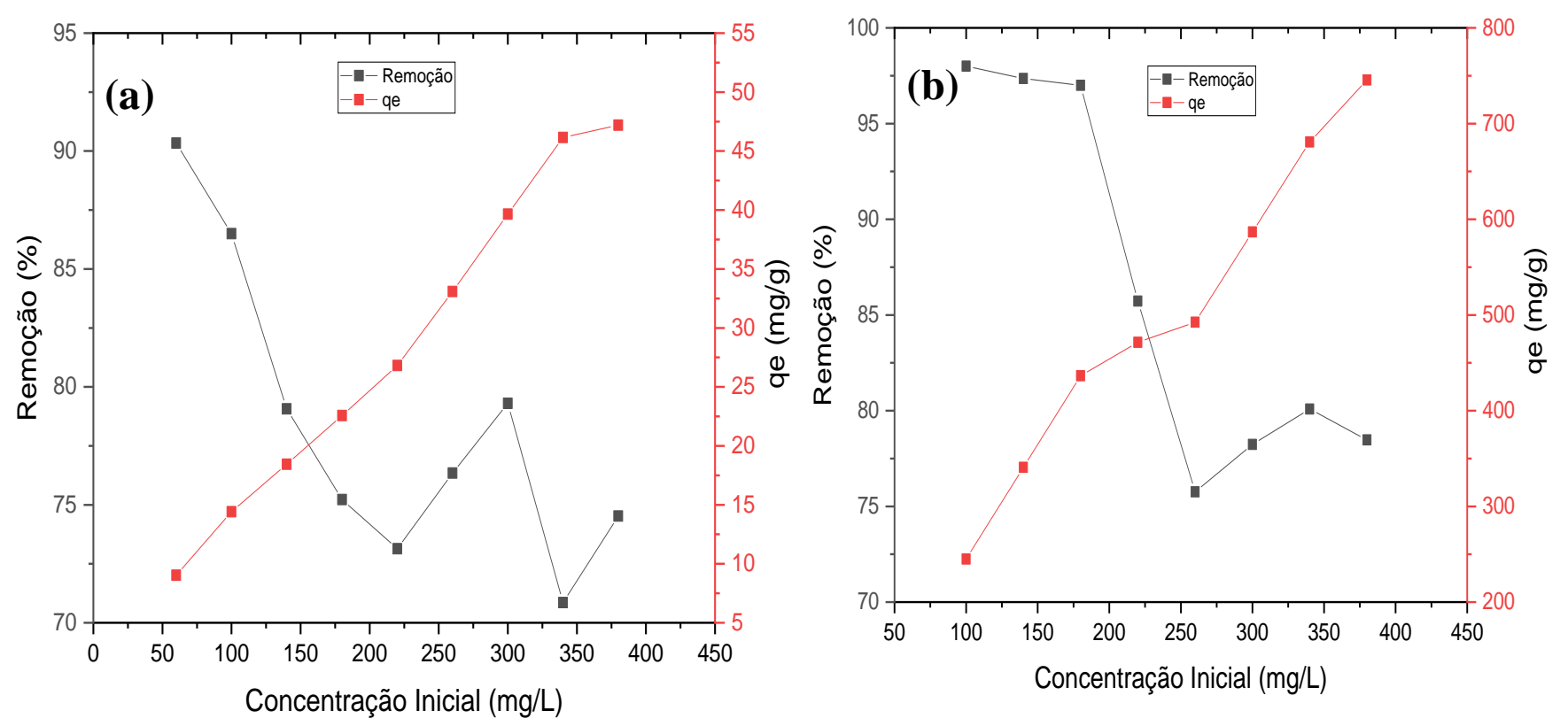

Fonte: Autores (2021).

É possível observar também ainda na Figura 14 que à medida que aumenta a concentração inicial aumenta o valor de $\mathrm{q}_{\mathrm{e}}$, segundo De Souza et al. (2019) isso acontece devido ao aumento do gradiente de massa entre a solução e o adsorvente, que atua como uma força motriz para a transferência das moléculas do corante da solução para a superfície do adsorvente. A Figura 15 mostra o efeito da variação de concentração inicial na capacidade de adsorção e na eficiência do processo de remoção.

Figura 15 - (a) Verde Malaquita 100 mg/L, (b) após adsorção com CAG-A, (c) após adsorção com CAG-B.

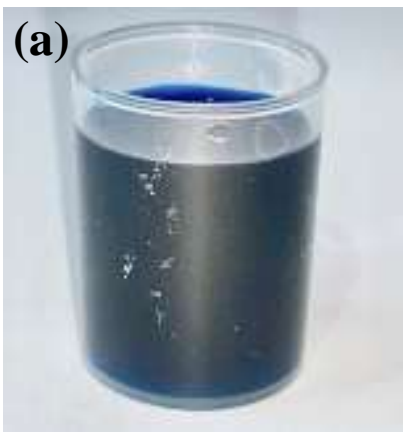

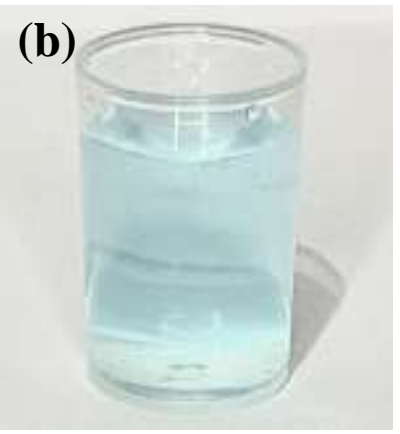

Fonte: Autores (2021).

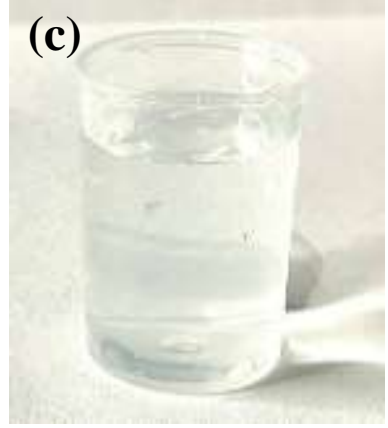


A porcentagem de remoção que decresce acentualmente pode não estar relacionada às concentrações iniciais ou ao aumento dessas, segundo Khan et al. (2012) e Vimonses et al. (2009) isso acontece porque à medida que quantidade de moléculas do Verde Malaquita aumenta, diminui os sítios disponíveis para a adsorção na sua superfície. Observa ainda que tanto CAG-A como CAG-B aumenta sua eficiência de remoção (no CAG-A a partir de $240 \mathrm{mg} / \mathrm{L}$ e no CAG-B a partir de $260 \mathrm{mg} / \mathrm{L}$ - Figura 14), podendo ser atribuída a saturação da primeira camada em sítios microporosos e início de novas camadas à medida que aumenta a concentração do corante.

\section{Conclusão}

Este estudo revelou que o caroço do açaí é um excelente material precursor para produção de carvão ativado, seja com hidróxido de sódio ou com ácido fosfórico. Pois em ambas as ativações químicas, as análises de caracterização mostraram que a superfície dos carvões obteve ótimas interações com o corante Verde Malaquita, em virtude dos grupos funcionais ácidos (mostrados pelo FTIR) e uma composição elementar que favoreceu a formação desses grupos funcionais (MEV/EDS), favorecendo o processo de adsorção como um todo. Além disso, foi observado através das curvas de ATG que os carvões apresentaram ótimos comportamentos térmicos. A alta eficiência na remoção do Verde Malaquita, sobretudo no processo adsortivo do CAG-B mostra que as condições experimentais em que se deu a adsorção foram satisfatórios

Com relação à cinética e ao equilíbrio de adsorção, os dados experimentais melhor se ajustaram, respectivamente, aos modelos de pseudo-segunda ordem e à isoterma de Freundlich tanto para as análises do CAG-B como para o CAG-A, sugerindo que o processo de adsorção ocorreu em multicamadas. Os valores máximos da capacidade de adsorção foram de $113,9 \mathrm{mg} / \mathrm{g} \mathrm{e}$ $668,88 \mathrm{mg} / \mathrm{g}$ para CAG-A e CAG-B respectivamente, comprovando que os adsorventes e as condições experimentais deste estudo, são ótimas opções para adsorver este corante.

Vale lembrar que esta pesquisa encontrou limitações na parte experimental sobretudo devido aos equipamentos de caracterização, mas especificamente na análise de área superficial específica, demonstrando uma baixa área superficial, que não condiz com a eficiência na remoção do corante estudado, sendo possível constatar que houve algum problema nesta análise, além disso o equipamento não consegue identificar microporos. Desta forma, recomenda-se que em futuros trabalhos, possam refazer a análise superficial específica para ambos os carvões em equipamento capaz de identificar microporosidade, depois sugere-se que seja estudado mais especificamente o fato da isoterma de adsorção de $\mathrm{N}_{2}$ apresentar adsorção negativa, verificando o que levou a um possível desprendimento do soluto em relação a superfície do adsorvente.

\section{Agradecimentos}

Em primeiro lugar eu quero agradecer a Deus pelo dom da minha vida e por providenciar tudo o que foi necessário para realizar esta pesquisa. Agradeço também aos meus pais Josué (in memorian) e Maria Luiza que sempre incentivaram-me e educaram-me da melhor forma possível dentro de suas possibilidades. Também quero deixar meus agradecimentos aos meus irmãos (Adriano e Adriana), cunhados (Johne e Carla) e meu sobrinho Enzo Gabriel que tanto contribuíram (direta ou indiretamente, consciente ou inconscientemente) para eu chegar onde estou. Minha sincera e infinita gratidão ao meu orientador Luiz Eduardo que muito ensinou-me, contribuindo para o meu aprendizado, crescimento pessoal e profissional.

Agradecer também ao meu coorientador João Rodrigo, a Universidade do Estado do Pará - UEPA, em especial aos coordenadores do CAMPUS VI (UEPA Paragominas), aos amigos Fernando e Gesivaldo, ao meu tio Márcio e minha tia Marina, minhas primas Sabrina e Bella pela nossa acolhida em Paragominas. Agradeço também aos meus irmãos de comunidade que tanto torceram e rezaram por mim durante todo esse período.

E por fim, mas não menos importante, agradeço às minhas diretoras Jádina Pontes e Helena Freire que sensibilizandose com a minha rotina acadêmica, manifestaram todo apoio possível e condições para que eu concluísse esse projeto. 


\section{Referências}

Ahmad, A. et al. (2015). Recent advances in new generation dye removal technologies: novel search for approaches to reprocess wastewater. RSC advances, 5(39), 30801-30818.

Ahmed, M. J., \& Dhedan, S. K. (2012). Equilibrium isotherms and kinetics modeling of methylene blue adsorption on agricultural wastes-based activated carbons. Fluid phase equilibria, 317, 9-14a.

Ahmed, M. J., \& Theydan, S. K. (2012). Physical and chemical characteristics of activated carbon prepared by pyrolysis of chemically treated date stones and its ability to adsorb organics. Powder Technology, 229, 237-245b.

Almeida, C. F. D. (2015). Produção de carvão ativado quimicamente a partir da palmácea mauritia flexuosa e o estudo de suas propriedades adsortivas.

Andrade, R. C. D. (2014). Preparação e caracterização de carvão ativado a partir de material alternativo lignocelulósico.

Ayawei, N., Ebelegi, A. N., \& Wankasi, D. (2017). Modelling and interpretation of adsorption isotherms. Journal of chemistry, 2017.

Azaman, S. H., Afandi, A., Hameed, B. H., \& Din, A. M. (2018). Removal of malachite green from aqueous phase using coconut shell activated carbon: Adsorption, desorption, and reusability studies. Journal of Applied Science and Engineering, 21(3), 317-330.

Azevedo, L. E. C. D. (2015). Adsorção de corantes básicos empregados na indústria têxtil por argila: cinética e perfil de equílibrio.

Bedin, K. C., Martins, A. C., Cazetta, A. L., Pezoti, O., \& Almeida, V. C. (2016). KOH-activated carbon prepared from sucrose spherical carbon: Adsorption equilibrium, kinetic and thermodynamic studies for Methylene Blue removal. Chemical Engineering Journal, 286, $476-484$.

Benadjemia, M., Millière, L., Reinert, L., Benderdouche, N., \& Duclaux, L. (2011). Preparation, characterization and Methylene Blue adsorption of phosphoric acid activated carbons from globe artichoke leaves. Fuel Processing Technology, 92(6), 1203-1212.

Bharti, V., Vikrant, K., Goswami, M., Tiwari, H., Sonwani, R. K., Lee, J., \& Singh, R. S. (2019). Biodegradation of methylene blue dye in a batch and continuous mode using biochar as packing media. Environmental research, 171, 356-364.

Biswas, S., \& Mishra, U. (2015). Effective remediation of lead ions from aqueous solution by chemically carbonized rubber wood sawdust: equilibrium, kinetics, and thermodynamic study. Journal of Chemistry, 2015.

Bulut, E., Özacar, M., \& Şengil, İ. A. (2008). Adsorption of malachite green onto bentonite: equilibrium and kinetic studies and process design. Microporous and mesoporous materials, 115(3), 234-246.

Calvete, T. et al. (2010). Application of carbon adsorbents prepared from Brazilian-pine fruit shell for the removal of reactive orange 16 from aqueous solution: kinetic, equilibrium, and thermodynamic studies. Journal of environmental management, 91(8), 1695-1706.

Cao, J. S., Lin, J. X., Fang, F., Zhang, M. T., \& Hu, Z. R. (2014). A new absorbent by modifying walnut shell for the removal of anionic dye: kinetic and thermodynamic studies. Bioresource technology, 163, 199-205.

Clark, H. L. M. (2010). Remoção de fenilalanina por adsorvente produzido a partir de torta prensada de grãos defeituosos de café.

Datta, D., Kerkez Kuyumcu, Ö., Bayazit, Ş. S., \& Abdel Salam, M. (2017). Adsorptive removal of malachite green and Rhodamine B dyes on Fe ${ }_{3} \mathrm{O}_{4} /$ activated carbon composite. Journal of Dispersion Science and Technology, 38(11), 1556-1562.

de Lima Yamaguchi, K. K., Pereira, L. F. R., Lamarão, C. V., Lima, E. S., \& da Veiga-Junior, V. F. (2015). Amazon acai: chemistry and biological activities: a review. Food chemistry, 179, 137-151.

De Marco, C. (2015). Preparação, caracterização e aplicação de um compósito ferromagnético na remoção do corante verde de malaquita em meio aquoso.

de Souza, T. N. V., de Carvalho, S. M. L., Vieira, M. G. A., da Silva, M. G. C., \& Brasil, D. D. S. B. (2018). Adsorption of basic dyes onto activated carbon: experimental and theoretical investigation of chemical reactivity of basic dyes using DFT-based descriptors. Applied Surface Science, 448, 662-670a.

de Souza, T. N. V., Vieira, M. G. A., da Silva, M. G. C., Brasil, D. D. S. B., \& de Carvalho, S. M. L. (2019). H ${ }_{3} \mathrm{PO}_{4}{ }^{-a}$ activated carbons produced from açai stones and Brazil nut shells: removal of basic blue 26 dye from aqueous solutions by adsorption. Environmental Science and Pollution Research, 26(28), 28533-28547b.

Fan, Z., Qi, D., Xiao, Y., Yan, J., \& Wei, T. (2013). One-step synthesis of biomass-derived porous carbon foam for high performance supercapacitors. Materials Letters, 101, 29-32.

Fernandes, M. R., Huang, X., Abbenhuis, H. C., \& Hensen, E. J. (2019). Lignin oxidation with an organic peroxide and subsequent aromatic ring opening. International journal of biological macromolecules, 123, 1044-1051.

Fiol, N., \& Villaescusa, I. (2009). Determination of sorbent point zero charge: usefulness in sorption studies. Environmental Chemistry Letters, 7(1), 79-84.

Galindo, L. S. G. (2012). Remoção de íons de chumbo e cádmio em diferentes sistemas de adsorção/troca iônica em argila bentonítica tipo Fluidgel.

Giannakoudakis, D. A., Hosseini-Bandegharaei, A., Tsafrakidou, P., Triantafyllidis, K. S., Kornaros, M., \& Anastopoulos, I. (2018). Aloe vera waste biomassbased adsorbents for the removal of aquatic pollutants: a review. Journal of environmental management, 227, 354-364.

Guimarães, I. D. S. (2006). Oxidação de carvões ativados de endocarpo de coco da baía com soluções de $\mathrm{HNO}_{3}$ e uma investigação sobre o método de Boehm. Universidade Federal da Paraíba, Departamento de Química, Jão Pessoa, PB, 81. 
El Haddad, M., Slimani, R., Mamouni, R., ElAntri, S., \& Lazar, S. (2013). Removal of two textile dyes from aqueous solutions onto calcined bones. Journal of the Association of Arab Universities for Basic and Applied Sciences, 14(1), 51-59.

Hao, J., Ji, L., Li, C., Hu, C., \& Wu, K. (2018). Rapid, efficient and economic removal of organic dyes and heavy metals from wastewater by zinc-induced insitu reduction and precipitation of graphene oxide. Journal of the Taiwan Institute of Chemical Engineers, 88, 137-145.

Hui, M., Shengyan, P., Yaqi, H., Rongxin, Z., Anatoly, Z., \& Wei, C. (2018). A highly efficient magnetic chitosan "fluid" adsorbent with a high capacity and fast adsorption kinetics for dyeing wastewater purification. Chemical Engineering Journal, 345, 556-565.

Instituto Brasileiro de Geografia e Estatística. (2020). Açaí. https://biblioteca.ibge.gov.br/visualizacao/periodicos/66/pam_2019_v46_br_informativo.pdf

Islam, M. A., Ahmed, M. J., Khanday, W. A., Asif, M., \& Hameed, B. H. (2017). Mesoporous activated carbon prepared from NaOH activation of rattan (Lacosperma secundiflorum) hydrochar for methylene blue removal. Ecotoxicology and environmental safety, 138, $279-285$.

Jain, S. N., Tamboli, S. R., Sutar, D. S., Jadhav, S. R., Marathe, J. V., Shaikh, A. A., \& Prajapati, A. A. (2020). Batch and continuous studies for adsorption of anionic dye onto waste tea residue: kinetic, equilibrium, breakthrough and reusability studies. Journal of Cleaner Production, $252,119778$.

Junior, O. P., et al. (2014). Synthesis of $\mathrm{ZnCl}_{2}$-activated carbon from macadamia nut endocarp (Macadamia integrifolia) by microwave-assisted pyrolysis: optimization using RSM and methylene blue adsorption. Journal of Analytical and Applied Pyrolysis, 105, $166-176$.

Kaouah, F., Boumaza, S., Berrama, T., Trari, M., \& Bendjama, Z. (2013). Preparation and characterization of activated carbon from wild olive cores (oleaster) by $\mathrm{H}_{3} \mathrm{PO}_{4}$ for the removal of Basic Red 46. Journal of Cleaner Production, 54, 296-306.

Katheresan, V., Kansedo, J., \& Lau, S. Y. (2018). Efficiency of various recent wastewater dye removal methods: a review. Journal of environmental chemical engineering, 6(4), 4676-4697.

Khan, T. A., Dahiya, S., Ali, I.. Use of kaolinite as adsorbent: Equilibrium, dynamics and thermodynamic studies on the adsorption of Rhodamine B from aqueous solution. Applied Clay Science, 69, 58-66, 2012.

Konicki, W., Cendrowski, K., Bazarko, G., \& Mijowska, E. (2015). Study on efficient removal of anionic, cationic and nonionic dyes from aqueous solutions by means of mesoporous carbon nanospheres with empty cavity. Chemical Engineering Research and Design, 94, $242-253$.

Köche, J. C. (2011). Fundamentos de metodologia científica. Editora Vozes.

Kumar, N., Mittal, H., Parashar, V., Ray, S. S., \& Ngila, J. C. (2016). Efficient removal of rhodamine 6G dye from aqueous solution using nickel sulphide incorporated polyacrylamide grafted gum karaya bionanocomposite hydrogel. RSC advances, 6(26), 21929-21939a.

Kumar, N., Reddy, L., Parashar, V., \& Ngila, J. C. (2017). Controlled synthesis of microsheets of ZnAl layered double hydroxides hexagonal nanoplates for efficient removal of Cr (VI) ions and anionic dye from water. Journal of environmental chemical engineering, 5(2), 1718-1731b.

Kumar, N., Fosso-Kankeu, E., \& Ray, S. S. (2019). Achieving controllable MoS2 nanostructures with increased interlayer spacing for efficient removal of Pb (II) from aquatic systems. ACS applied materials \& interfaces, 11(21), 19141-19155c.

Kundu, S., \& Gupta, A. K. (2006). Arsenic adsorption onto iron oxide-coated cement (IOCC): regression analysis of equilibrium data with several isotherm models and their optimization. Chemical Engineering Journal, 122(1-2), 93-106.

Lucena, L. M. D. (2018). Estudo do processo de adsorção com o carvão ativado proveniente do endocarpo de coco (Coccus nucifera L.) para remoção de cor, $D Q O$ e toxicidade de efluente têxtil (Master's thesis, Universidade Federal de Pernambuco).

Mangueira, E. S. V. (2014). Produção de carvão ativado a partir de endocarpo de coco da baía (Cocos nucifera) aplicado ao processo de adsorção do herbicida metribuzin.

Marinho, S. B. N., de Oliveira, J. D. M., do Nascimento Gomes, D., Rocha, B. S., Yara, R., de Andrade Lima, C. S., \& Machado, D. C. (2019). Caracterização de scaffold poroso obtido pela técnica de freezecasting. Anais do III Simpósio de Inovação em Engenharia Biomédica-SABIO $2019,43$.

Masoumi, S., \& Dalai, A. K. (2020). Optimized production and characterization of highly porous activated carbon from algal-derived hydrochar. Journal of Cleaner Production, 263, 121427.

Mittal, H., \& Mishra, S. B. (2014). Gum ghatti and Fe3O4 magnetic nanoparticles based nanocomposites for the effective adsorption of rhodamine B. Carbohydrate polymers, 101, 1255-1264.

Mohammad, M., Maitra, S., \& Dutta, B. K. (2018). Comparison of activated carbon and physic seed hull for the removal of malachite green dye from aqueous solution. Water, Air, \& Soil Pollution, 229(2), 1-14.

Murthy, T. K., Gowrishankar, B. S., Prabha, M. C., Kruthi, M., \& Krishna, R. H. (2019). Studies on batch adsorptive removal of malachite green from synthetic wastewater using acid treated coffee husk: equilibrium, kinetics and thermodynamic studies. Microchemical Journal, 146, $192-201$.

Muttakin, M., Mitra, S., Thu, K., Ito, K., \& Saha, B. B. (2018). Theoretical framework to evaluate minimum desorption temperature for IUPAC classified adsorption isotherms. International Journal of Heat and Mass Transfer, 122, 795-805.

Nascimento, R. F. D., Lima, A. C. A. D., Vidal, C. B., Melo, D. D. Q., \& Raulino, G. S. C. (2014). Adsorção: aspectos teóricos e aplicações ambientais.

Nascimento, B. F. D. (2019). Adsorção de furfural em carvão ativado do endocarpo de açaí (Master's thesis, Universidade Federal de Pernambuco).

Naushad, M., ALOthman, Z. A., \& Javadian, H. (2015). Removal of Pb (II) from aqueous solution using ethylene diamine tetra acetic acid-Zr (IV) iodate composite cation exchanger: kinetics, isotherms and thermodynamic studies. Journal of Industrial and Engineering Chemistry, $25,35-41$. 
Nazari, M., \& Halladj, R. (2014). Adsorptive removal of fluoride ions from aqueous solution by using sonochemically synthesized nanomagnesia/alumina adsorbents: an experimental and modeling study. Journal of the Taiwan Institute of Chemical Engineers, 45(5), 2518-2525.

Noorimotlagh, Z., Soltani, R. D. C., Khataee, A. R., Shahriyar, S., \& Nourmoradi, H. (2014). Adsorption of a textile dye in aqueous phase using mesoporous activated carbon prepared from Iranian milk vetch. Journal of the Taiwan Institute of Chemical Engineers, 45(4), $1783-1791$.

Ofomaja, A. E., \& Ho, Y. S. (2007). Effect of pH on cadmium biosorption by coconut copra meal. Journal of Hazardous Materials, 139(2), 356-362.

Oliveira, R. F. (2013). Estudo da adsorção de cromo hexavalente em altas concentrações.

Oliveira, L. D., \& Tavares, G. D. S. (2016). Programa de desenvolvimento da cadeia produtiva do açaí no Estado do Pará (PRO-AÇAÍ). Belém, Pará, SEDAP.

Pallarés, J., González-Cencerrado, A., \& Arauzo, I. (2018). Production and characterization of activated carbon from barley straw by physical activation with carbon dioxide and steam. Biomass and Bioenergy, 115, 64-73.

Pereira, A. S., Shitsuka, D. M., Parreira, F. J., \& Shitsuka, R. (2018). Metodologia da pesquisa científica. UFSM.

Pereira, R. G., Veloso, C. M., da Silva, N. M., de Sousa, L. F., Bonomo, R. C. F., de Souza, A. O., \& Fontan, R. D. C. I. (2014). Preparation of activated carbons from cocoa shells and siriguela seeds using $\mathrm{H}_{3} \mathrm{PO}_{4}$ and $\mathrm{ZnCl}_{2}$ as activating agents for BSA and $\alpha$-lactalbumin adsorption. Fuel Processing Technology, 126, 476486.

Porpino, K. K. P. (2009). Biossorção de ferro (II) por casca de caranguejo Ucides Cordatus (Doctoral dissertation, Dissertação (Mestrado em Química)Universidade Federal da Paraíba, João Pessoa).

Prola, L. D., Machado, F. M., Bergmann, C. P., de Souza, F. E., Gally, C. R., Lima, E. C., \& Calvete, T. (2013). Adsorption of Direct Blue 53 dye from aqueous solutions by multi-walled carbon nanotubes and activated carbon. Journal of environmental management, 130, 166-175.

Puziy, A. M., Poddubnaya, O. I., Martínez-Alonso, A., Castro-Muñiz, A., Suárez-García, F., \& Tascón, J. M. (2007). Oxygen and phosphorus enriched carbons from lignocellulosic material. Carbon, 45(10), 1941-1950.

Ray, S. S.; Gusain, R; Kuma, N. (2020) Adsorption equilibrium isotherms, kinetics and thermodynamics. Elsevier.

Regti, A., Laamari, M. R., Stiriba, S. E., \& El Haddad, M. (2017). Use of response factorial design for process optimization of basic dye adsorption onto activated carbon derived from Persea species. Microchemical Journal, 130, 129-136.

Reis, D. C. N. S. P. (2013). Importância do estudo da área superficial específica e porosidade do estearato de magnésio para o setor farmacêutico.

Rimoli, M. F. D. S., Nogueira, R. M., Ferrarini, S. R., Castro, P. M. D., \& Pires, E. M. (2019). Preparation and characterization of carbon from the fruit of Brazil nut tree activated by physical process. Revista Árvore, 43(2).

Royer, B., Cardoso, N. F., Lima, E. C., Macedo, T. R., \& Airoldi, C. (2010). A useful organofunctionalized layered silicate for textile dye removal. Journal of hazardous materials, 181(1-3), 366-374.

Saleh, T. A., Sulaiman, K. O., AL-Hammadi, S. A., Dafalla, H., \& Danmaliki, G. I. (2017). Adsorptive desulfurization of thiophene, benzothiophene and dibenzothiophene over activated carbon manganese oxide nanocomposite: with column system evaluation. Journal of Cleaner Production, 154, 401-412a.

Saleh, T. A., Adio, S. O., Asif, M., \& Dafalla, H. (2018). Statistical analysis of phenols adsorption on diethylenetriamine-modified activated carbon. Journal of Cleaner Production, 182, 960-968b.

Salima, A., Benaouda, B., Noureddine, B., \& Duclaux, L. (2013). Application of Ulva lactuca and Systoceira stricta algae-based activated carbons to hazardous cationic dyes removal from industrial effluents. Water research, 47(10), 3375-3388.

Sato, M. K. (2018). Biocarvão de resíduos de açaí como condicionante de solos.

Santhi, T., Manonmani, S., \& Smitha, T. (2010). Removal of malachite green from aqueous solution by activated carbon prepared from the epicarp of Ricinus communis by adsorption. Journal of hazardous materials, 179(1-3), 178-186.

Santhosh, C., Velmurugan, V., Jacob, G., Jeong, S. K., Grace, A. N., \& Bhatnagar, A. (2016). Role of nanomaterials in water treatment applications: a review. Chemical Engineering Journal, 306, 1116-1137.

Schultz, J. (2012). Obtenção de carvão ativado a partir de resíduos agroindustriais para adsorção de antibiótico-amoxicilina.

Shamsuddin, M. S., Yusoff, N. R. N., \& Sulaiman, M. A. (2016). Synthesis and characterization of activated carbon produced from kenaf core fiber using $\mathrm{H}_{3} \mathrm{PO}_{4}$ activation. Procedia Chemistry, 19, 558-565.

Slimani, R., Anouzla, A., Abrouki, Y., Ramli, Y., El Antri, S., Mamouni, R., ... \& El Haddad, M. (2011). Removal of a cationic dye-Methylene Blue-from aqueous media by the use of animal bone meal as a new low cost adsorbent. J. Mater. Environ. Sci, 2(1), 77-87.

Tan, K. L., \& Hameed, B. H. (2017). Insight into the adsorption kinetics models for the removal of contaminants from aqueous solutions. Journal of the Taiwan Institute of Chemical Engineers, 74, 25-48.

Tang, Y. B., Liu, Q., \& Chen, F. Y. (2012). Preparation and characterization of activated carbon from waste ramulus mori. Chemical Engineering Journal, 203, $19-24$.

Vincent, T., Taulemesse, J. M., Dauvergne, A., Chanut, T., Testa, F., \& Guibal, E. (2014). Thallium (I) sorption using Prussian blue immobilized in alginate capsules. Carbohydrate polymers, 99, 517-526. 
Research, Society and Development, v. 10, n. 2, e49110212871, 2021 (CC BY 4.0) | ISSN 2525-3409 | DOI: http://dx.doi.org/10.33448/rsd-v10i2.12871

Vikrant, K., Giri, B. S., Raza, N., Roy, K., Kim, K. H., Rai, B. N., \& Singh, R. S. (2018). Recent advancements in bioremediation of dye: current status and challenges. Bioresource technology, 253, 355-367.

Vimonses, V., Lei, S., Jin, B., Chow, C. W., \& Saint, C. (2009). Adsorption of congo red by three Australian kaolins. Applied clay science, 43(3-4), 465-472.

Xu, M. C., Zhou, Y., \& Huang, J. H. (2008). Adsorption behaviors of three polymeric adsorbents with amide groups for phenol in aqueous solution. Journal of colloid and interface science, 327(1), 9-14.

Xu, J., Chen, L., Qu, H., Jiao, Y., Xie, J., \& Xing, G. (2014). Preparation and characterization of activated carbon from reedy grass leaves by chemical activation with $\mathrm{H}_{3} \mathrm{PO}_{4}$. Applied Surface Science, 320, 674-680.

Yan, H., Yang, L., Yang, Z., Yang, H., Li, A., \& Cheng, R. (2012). Preparation of chitosan/poly (acrylic acid) magnetic composite microspheres and applications in the removal of copper (II) ions from aqueous solutions. Journal of hazardous materials, 229, 371-380.

Yorgun, S., \& Yıldiz, D. (2015). Preparation and characterization of activated carbons from Paulownia wood by chemical activation with $\mathrm{H}_{3} \mathrm{PO}_{4}$. Journal of the Taiwan Institute of Chemical Engineers, 53, 122-131.

Yousef, R. I., El-Eswed, B., \& Ala'a, H. (2011). Adsorption characteristics of natural zeolites as solid adsorbents for phenol removal from aqueous solutions: kinetics, mechanism, and thermodynamics studies. Chemical engineering journal, 171(3), 1143-1149.

Yu, M., Han, Y., Li, J., \& Wang, L. (2017). $\mathrm{CO}_{2}$-activated porous carbon derived from cattail biomass for removal of malachite green dye and application as supercapacitors. Chemical Engineering Journal, 317, 493-502.

Zhang, J., Li, Y., Zhang, C., \& Jing, Y. (2008). Adsorption of malachite green from aqueous solution onto carbon prepared from Arundo donax root. Journal of hazardous materials, 150(3), 774-782.

Zhang, Y., Song, X., Xu, Y., Shen, H., Kong, X., \& Xu, H. (2019). Utilization of wheat bran for producing activated carbon with high specific surface area via $\mathrm{NaOH}$ activation using industrial furnace. Journal of cleaner production, 210, 366-375.

Zhou, L., Zhou, H., Hu, Y., Yan, S., \& Yang, J. (2019). Adsorption removal of cationic dyes from aqueous solutions using ceramic adsorbents prepared from industrial waste coal gangue. Journal of environmental management, 234, 245-252. 